\title{
Robust H-Infinity Stabilization and Resilient Filtering for Discrete-Time Constrained Singular Piecewise-Affine Systems
}

\author{
Zhenhua Zhou, Mao Wang, and Qitian Yin \\ Space Control and Inertial Technology Research Center, Harbin Institute of Technology, Harbin 150080, China \\ Correspondence should be addressed to Zhenhua Zhou; zhouzhenhuazzh@163.com
}

Received 7 June 2014; Accepted 12 November 2014

Academic Editor: Asier Ibeas

Copyright (C) 2015 Zhenhua Zhou et al. This is an open access article distributed under the Creative Commons Attribution License, which permits unrestricted use, distribution, and reproduction in any medium, provided the original work is properly cited.

\begin{abstract}
This paper is concerned with the problem of designing robust $\mathrm{H}$-infinity output feedback controller and resilient filtering for a class of discrete-time singular piecewise-affine systems with input saturation and state constraints. Based on a singular piecewise Lyapunov function combined with S-procedure and some matrix inequality convexifying techniques, the $\mathrm{H}$-infinity stabilization condition is established and the resilient $\mathrm{H}$-infinity filtering error dynamic system is investigated, and, meanwhile, the domain of attraction is well estimated. Under energy bounded disturbance, the input saturation disturbance tolerance condition is proposed; then, the resilient $\mathrm{H}$-infinity filter is designed in some restricted region. It is shown that the controller gains and filter design parameters can be obtained by solving a family of LMIs parameterized by one or two scalar variables. Meanwhile, by using the corresponding optimization methods, the domain of attraction and the disturbance tolerance level is maximized, and the $\mathrm{H}$-infinity performance $\gamma$ is minimized. Numerical examples are given to illustrate the effectiveness of the proposed design methods.
\end{abstract}

\section{Introduction}

Piecewise-affine systems offer a good modeling framework for hybrid systems involving nonlinear phenomena which have the characteristic of both continuous dynamics and discrete events with the nature of the model switching [1-5]. Piecewise-affine systems which are composed of a partition of the state space and local dynamics valid can describe a rich class of practical circuits and control systems when some nonlinear components are encountered, such as saturation, dead-zone, and relays [6-8]. In fact, many nonlinearities that appear frequently in engineering systems either are piecewise-affine or can be approximated as piecewise-affine functions, which can be used to analyze smooth nonlinear systems with arbitrary accuracy $[9,10]$.

Robust stabilization problems of piecewise-affine systems with norm-bounded time-varying parameters uncertainties have been extensively studied, and various results have been obtained on the analysis and controller synthesis [11]. To mention a few, the problem of well-posedness was investigated as a basic issue for piecewise-affine systems in the literature [12]. By presenting a number of algorithms, the authors in [6] tested the controllability and observability of piecewise-affine systems. In $[9,10]$, more attention was paid by constructing a piecewise-affine Lyapunov function on stability and optimal performance analysis for piecewiseaffine system. By the same Lyapunov functions as in [9], controller synthesis and state estimation of piecewise-affine system were considered in [13-16]. By using a common Lyapunov function and a piecewise Lyapunov function, respectively, the authors in [17-23] investigated the analysis and control of systems that may involve multiple equilibrium points. Using a similar method to that in [10-12], some results have also been reported in [24-26] where the piecewise Lyapunov function might be discontinuous across the region boundaries. Recently, much more attention has been paid to the problem that the stabilization conditions can be determined by solving a set of linear matrix inequalities (LMIs). A number of results have been reported based on the piecewise Lyapunov function [27-30], such as controller synthesis, state estimation, output regulation, and tracking of piecewise-affine system. For a filtering error dynamic system, the objective of filter designing is to estimate the unavailable state variables. During the past decades, much more filtering 


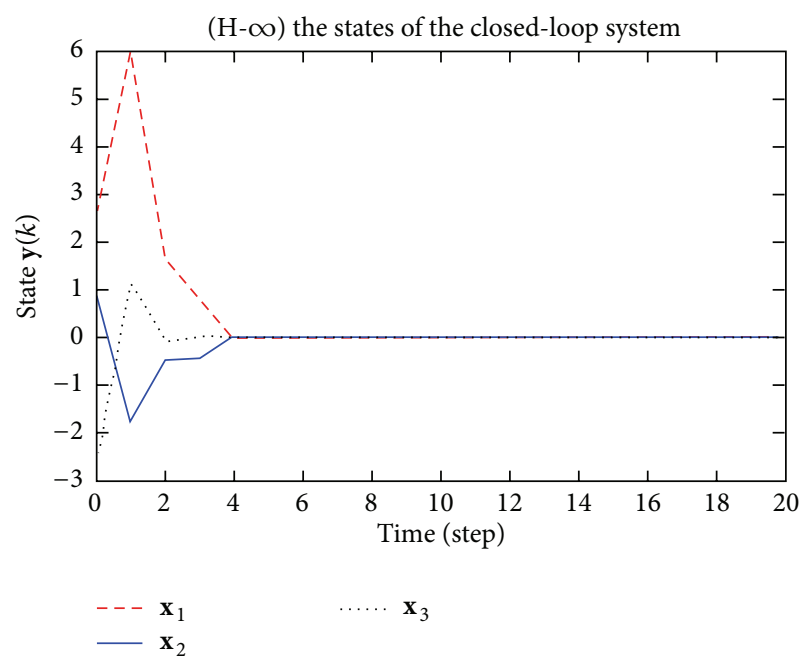

FIGURE 1: State responses of the closed-loop system.

schemes have been investigated, such as Kalman filtering, $\mathrm{H}-$ infinity filtering, and reduced-order $\mathrm{H}$-infinity filtering [3134]. Then, the authors in [35] paid more attention to the problem of resilient Kalman filtering with respect to estimator gain perturbations. And the resilient $\mathrm{H}$-infinity filtering was also raised.

For output feedback control systems, an actuator with amplitude and rate limitations may be considered in most real-world applications that often suffer from the state constraints. For controller synthesis, ignoring these constraints may degrade the performance and may even cause the instability of closed-loop system [36, 37] (Figure 1). On the other hand, output feedback control can be easily implemented with low cost, which is particularly very useful and more realistic [38]. In the literature [39], the low gain feedback designs were investigated for linear systems with all of its open loop poles in the closed left-half plane. Based on an auxiliary feedback matrix, the authors in $[40,41]$ studied the stabilization and $L_{2}$-gain control of piecewise-linear systems with actuator saturation. Recently, the authors in $[42,43]$ investigated state feedback $\mathrm{H}$-infinity control and output feedback $\mathrm{H}$-infinity control for piecewise-affine systems, respectively.

In this paper, the $\mathrm{H}$-infinity output feedback control and resilient filter design problems of singular piecewiseaffine systems with input saturation and state constraints are considered. Based on a singular piecewise Lyapunov function combined with S-procedure and some matrix inequality convexifying techniques, the $\mathrm{H}$-infinity stabilization condition is established and the resilient $\mathrm{H}$-infinity filtering error dynamic system is investigated. Under energy bounded disturbance, the input saturation disturbance tolerance condition is proposed; then, the resilient $\mathrm{H}$-infinity filter is designed in some restricted region. The results are given in terms of solutions to a set of linear matrix inequalities. Meanwhile, by using the corresponding optimization methods, the domain of attraction and the disturbance tolerance level is maximized, and the $\mathrm{H}$-infinity performance $\gamma$ is minimized.
According to the existing results, the main contributions of this paper can be summarized as follows: (1) by adding a resilient block to output feedback controller, the robust stabilization of resilient $\mathrm{H}$-infinity output feedback closed-loop control systems is investigated, which was not considered in [44] (Figure 3); (2) for singular piecewise-affine systems, the analysis and maximization of the disturbance tolerance capability are considered; (3) by investigating resilient $\mathrm{H}$ infinity filter, the robust stabilization of resilient filtering error dynamic system is considered for the first time.

The paper is organized as follows. In Section 2, model description and some preliminaries are given. Sufficient conditions for designing robust $\mathrm{H}$-infinity output feedback controllers are proposed in Section 3 firstly; then, a resilient $\mathrm{H}$-infinity filter is given by using the analysis and synthesis methods described previously; the resulting filtering error dynamic system is admissible with the resilient $\mathrm{H}$-infinity filter. Two numerical examples are presented to illustrate the effectiveness of the proposed approaches in Section 4, which is followed by some conclusions finally.

Notation 1. The notations used throughout the paper are standard. $\mathbf{R}^{n}$ denotes the $n$-dimensional Euclidean space, while $\mathbf{R}^{m \times n}$ refers to the set of all real matrices with $m$ rows and $n$ columns. $\mathbf{A}^{T}$ represents the transpose of the matrix $\mathbf{A}$, while $\mathbf{A}^{-1}$ denotes the inverse matrix of $\mathbf{A} . \mathbf{l}_{2}[0, \infty)$ refers to the space of square summable infinite sequences with the Euclidean norm $\|\cdot\| . \mathbf{I}$ is the identity matrix with appropriate dimensions. For real symmetric matrices $\mathbf{X}$ and $\mathbf{Y}$, the notation $\mathbf{X} \geq \mathbf{Y}$ (resp., $\mathbf{X}>\mathbf{Y}$ ) means that the matrix $\mathbf{X}-\mathbf{Y}$ is positive semidefinite (resp., positive definite). The notation ${ }^{*}$ is used to indicate the terms that can be induced by symmetry. $[1, \hbar]$ denotes the set of $1,2, \ldots, \hbar$, in which the elements are integers.

\section{Model Description and Problem Formulation}

Consider a discrete-time singular piecewise-affine system with norm-bounded uncertainties and input saturation described by the following dynamics:

$$
\begin{gathered}
\mathbf{E x}(k+1)=\left(\mathbf{A}_{i}+\Delta \mathbf{A}_{i}\right) \mathbf{x}(k)+\mathbf{B}_{i} \operatorname{sat}(\mathbf{u}(k)) \\
+\mathbf{D}_{i 1} \mathbf{w}(k)+\mathbf{E}\left(\mathbf{b}_{i}+\Delta \mathbf{b}_{i}\right), \\
\mathbf{y}(k)=\mathbf{C}_{i} \mathbf{x}(k), \\
\mathbf{z}(k)=\mathbf{F}_{i} \mathbf{x}(k)+\mathbf{G}_{i} \operatorname{sat}(\mathbf{u}(k))+\mathbf{D}_{i 2} \mathbf{w}(k), \\
\mathbf{x}(k), \mathbf{y}(k) \in \mathfrak{R}_{i}, \quad i \in I, \mathbf{x}(0)=\mathbf{x}_{0},
\end{gathered}
$$

where $\mathbf{x}(k) \in \mathbf{R}^{n_{x}}$ is the system state vector, $\mathbf{u}(k) \in \mathbf{R}^{n_{u}}$ is the system control input, $\mathbf{y}(k) \in \mathbf{R}^{n_{y}}$ is the system measurement output vector, $\mathbf{z}(k) \in \mathbf{R}^{n_{z}}$ is the controlled output vector, and $\mathbf{w}(k) \in \mathbf{R}^{n_{w}}$ is an energy bounded disturbance input which belongs to $\mathbf{l}_{2}[0, \infty)$ and satisfies $\sum_{t=0}^{\infty} \mathbf{w}^{T}(t) \mathbf{w}(t) \leq \beta<\infty$. $\mathbf{A}_{i}, \mathbf{B}_{i}, \mathbf{C}_{i}, \mathbf{D}_{i 1}, \mathbf{D}_{i 2}, \mathbf{F}_{i}, \mathbf{G}_{i}, \mathbf{b}_{i}$, and $\mathbf{E}$ are known real constant matrices with appropriate dimensions, denoting the $i$ th local model of the system, and $\mathbf{E} \mathbf{b}_{i}$ is the offset term. The index set 
of cells is denoted by $I=\{1,2, \ldots, N\}$. The matrix $\mathbf{E} \in \mathbf{R}^{n_{x} \times n_{x}}$ may be singular and $\operatorname{rank}(\mathbf{E})=r \leq n_{x}$ is assumed. $\Delta \mathbf{A}_{i}$ and $\Delta \mathbf{b}_{i}$ are real matrices representing parameter uncertainties of the $i$ th local model of the system, which are assumed to be norm-bounded as

$$
\left[\begin{array}{ll}
\Delta \mathbf{A}_{i} & \mathbf{E} \Delta \mathbf{b}_{i}
\end{array}\right]=\mathbf{W}_{i 1} \Delta_{i}(t)\left[\begin{array}{ll}
\mathbf{E}_{i 1} & \mathbf{E}_{i 2}
\end{array}\right], \quad i \in I,
$$

where $\mathbf{W}_{i 1}, \mathbf{E}_{i 1}$, and $\mathbf{E}_{i 2}$ are known real constant matrices with appropriate dimensions. $\Delta_{i}(k): Z^{+} \rightarrow \mathbf{R}^{s_{1} \times s_{2}}$ is an unknown real-valued time-varying matrix function with the Lebesgue measurable elements satisfying

$$
\Delta_{i}^{T}(k) \Delta_{i}(k) \leq \mathbf{I}_{s_{2}} .
$$

The parameter uncertainties are said to be admissible if (3)(4) hold.

Remark 1. In order to refrain unnecessarily complicated notations, in this paper, we only consider the norm-bounded uncertainty parameters involving matrices $\mathbf{A}_{i}$ and $\mathbf{b}_{i}$. Nevertheless, the methods to be investigated in this paper can be easily extended to the case when the uncertainty parameters also emerge in the matrices $\mathbf{B}_{i}, \mathbf{D}_{i 1}, \mathbf{D}_{i 2}, \mathbf{F}_{i}$, and $\mathbf{G}_{i}, i \in I$.

In addition, we consider that the system is subject to the input and state constraints, where the saturated input is expressed by $\operatorname{sat}(\mathbf{u}(k))=$ $\left[\operatorname{sat}\left(\mathbf{u}_{1}(k)\right) \operatorname{sat}\left(\mathbf{u}_{2}(k)\right) \cdots \operatorname{sat}\left(\mathbf{u}_{m}(k)\right)\right]^{T}, \quad \operatorname{sat}\left(\mathbf{u}_{l}\right)=$ $\operatorname{sgn}\left(\mathbf{u}_{l}\right) \min \left\{\left|\mathbf{u}_{l}\right|, \overline{\mathbf{u}}\right\}, l \in[1, m]$, with saturation level $\overline{\mathbf{u}}$, and the system state is bounded by the following condition:

$$
-\overline{\mathbf{g}} \leq \mathbf{L x} \leq \overline{\mathbf{g}}
$$

where $\mathbf{L} \in \mathbf{R}^{n_{r} \times n_{x}}$ is a known real constant matrix and $\overline{\mathbf{g}} \in \mathbf{R}^{n_{r}}$ is a given constant vector.

In the following, we will introduce a new set:

$$
\Omega=\left\{(i, j) \mid \mathbf{y}(k) \in \mathfrak{R}_{i}, \mathbf{y}(k+1) \in \mathfrak{R}_{j}, i, j \in I\right\}
$$

which represents the index pairs denoting all possible transitions of the system state trajectories.

Remark 2. For mixed logical dynamical (MLD) system [6], the reachability analysis can usually determine the set $\Omega$. If it is probable that the transitions happen between all regions, then $\Omega:=I \times I=\{(i, j) \mid i, j \in I\}$.

It is assumed in this paper that the polyhedral region $\mathfrak{R}_{i}, i \in I$, is slabs of the following form:

$$
\mathfrak{R}_{i}=\left\{\mathbf{y} \mid \alpha_{i} \leq \mathbf{y} \leq \beta_{i}, \mathbf{y}=\mathbf{C}_{i} \mathbf{x}\right\}, \quad i \in I .
$$

Each slab can be exactly described by a degenerate ellipsoid:

$$
\varepsilon_{i}=\left\{\mathbf{x} \mid\left\|\overline{\mathbf{F}}_{i} \mathbf{x}+f_{i}\right\| \leq 1\right\}, \quad i \in I,
$$

where $\overline{\mathbf{F}}_{i}=2 \mathbf{C}_{i} /\left(\beta_{i}-\alpha_{i}\right), f_{i}=-\left(\beta_{i}+\alpha_{i}\right) /\left(\beta_{i}-\alpha_{i}\right)$. Then we have the following relationship for each ellipsoid region:

$$
\left[\begin{array}{c}
\mathbf{x}(k) \\
1
\end{array}\right]^{T}\left[\begin{array}{cc}
\overline{\mathbf{F}}_{i}^{T} \overline{\mathbf{F}}_{i} & \overline{\mathbf{F}}_{i}^{T} f_{i} \\
* & f_{i}^{T} f_{i}-1
\end{array}\right]\left[\begin{array}{c}
\mathbf{x}(k) \\
1
\end{array}\right] \leq 0, \quad i \in I .
$$

Let the partitioned regions be separated into two classes $I=I_{0} \cup I_{1}$, where $I_{0}$ denotes the index set of regions with $f_{i}^{T} f_{i}-1 \leq 0$ which contains the origin and $I_{1}$ denotes the index set of regions otherwise.

For system (1), we introduce the following definitions.

Definition 3 (see [45]). According to the discrete-time piecewise-affine singular system (1) with $\mathbf{u}(k)=0$, one has the following.

(i) The system is said to be regular if $\operatorname{det}\left(\mathbf{z E}-\mathbf{A}_{i}\right)$ is not identically zero, $i \in I$.

(ii) The system is said to be causal if $\operatorname{deg}\left(\operatorname{det}\left(\mathbf{z E}-\mathbf{A}_{i}\right)\right)=$ $\operatorname{rank}(\mathbf{E}), i \in I$.

(iii) The system is said to be stable if all roots of $\lambda\left(\mathbf{E}, \mathbf{A}_{i}\right) \subset$ $\mathbf{D}_{\text {int }}(0,1)$.

(iv) The system is said to be admissible if it is regular, causal, and stable.

(v) For the system, there exists a grade 1 (infinite generalised) eigenvector of the pair ( $\left.\mathbf{E}, \mathbf{A}_{i}\right)$, such that for any nonzero vector $\boldsymbol{\nu}^{1}$ satisfying $\mathbf{E} \boldsymbol{\nu}^{1}=0$, and there exists a grade $k$ (infinite generalised) eigenvector of the pair $\left(\mathbf{E}, \mathbf{A}_{i}\right)$, such that for any nonzero vector $\boldsymbol{\nu}^{k}(k \geq 2)$ satisfying $\mathbf{E} \nu^{k}=\mathbf{A}_{i} \nu^{k-1}$.

Definition 4. For the convenience of the subsequent descriptions, one defines the sets $\boldsymbol{\varepsilon}(\mathbf{P}, \Upsilon)=\left\{\mathbf{x}(t) \mid \mathbf{x}^{T}(t) \mathbf{P x}(t) \leq\right.$ $\Upsilon, \Upsilon>0\}$ and $\zeta(\mathbf{H}, \tau)=\left\{\mathbf{x}(t)|| \mathbf{H}_{l} \mathbf{x}(t) \mid \leq \tau, \tau>0\right\}$ as an ellipsoid and a polyhedron, where $l \in[1, m]$, respectively, $\mathbf{P}$ is a positive definite matrix, and $\mathbf{H}_{l}$ is the $l$ th row of the matrix $\mathbf{H} \in \mathbf{R}^{n_{u} \times n_{x}}$. Also, one introduces the following lemmas.

Lemma 5 (see [46]). For all $\mathbf{u} \in \mathbf{R}^{n_{u}}$ and $\boldsymbol{v} \in \mathbf{R}^{n_{u}}$ such that $\left|\boldsymbol{\nu}_{l}\right|<\overline{\mathbf{u}}, l \in[1, m]$, one has $\operatorname{sat}(\mathbf{u}) \in \operatorname{co}\left\{\mathbf{w}_{s} \mathbf{u}+\overline{\mathbf{w}_{s}} \boldsymbol{\nu}, s \in\right.$ $\left.\left[1,2^{m}\right]\right\}$, where "co" denotes the convex hull. Here, $\mathbf{w}_{s}$ is an $n_{u} \times$ $n_{u}$ diagonal matrix with elements either 1 or 0 . On the other hand, $\overline{\mathbf{w}_{s}}=\mathbf{I}-\mathbf{w}_{s}$. There are $2^{m}$ such matrices.

Lemma 6 (see [46]). Let matrices $\mathbf{M}=\mathbf{M}^{T}, \mathbf{S}, \mathbf{N}$, and $\Delta(t)$ be real matrices of appropriate dimensions and the inequality, $\mathbf{M}+\mathbf{S} \Delta(t) \mathbf{N}+\mathbf{N}^{T} \Delta^{T}(t) \mathbf{S}^{T}<0$, holds for all $\Delta^{T}(t) \Delta(t) \leq I$ if and only if for some positive scalar $\varepsilon>0$ such that $\mathbf{M}+\varepsilon \mathbf{S S}^{T}+$ $\varepsilon^{-1} \mathbf{N}^{T} \mathbf{N}<0$.

(A) Resilient Output Feedback Control of the Discrete-Time Singular Piecewise-Affine System. In this paper, we consider a resilient output feedback controller which is described as follows:

$$
\begin{array}{r}
\mathbf{u}(k)=\left(\mathbf{K}_{i}+\Delta \mathbf{K}_{i}\right) \mathbf{y}(k), \quad \mathbf{K}_{i} \in \mathbf{R}^{n_{u} \times n_{y}}, \\
\mathbf{y}(k) \in \mathfrak{R}_{i}, \quad i \in \mathbf{I},
\end{array}
$$

where $\Delta \mathbf{K}_{i}=\mathbf{W}_{i 1} \Delta_{i}(t) \mathbf{E}_{i 3}, i \in I$, and $\mathbf{E}_{i 3}$ is known real constant matrices with appropriate dimensions.

In this paper, for all $\mathbf{u} \in \mathbf{R}^{n_{u}}$ and $\boldsymbol{v} \in \mathbf{R}^{n_{u}}$ such that $\left|\boldsymbol{v}_{l}\right|<$ $\overline{\mathbf{u}}, l \in[1, m]$, we assume that $\mathbf{x}(k) \in \zeta\left(\mathbf{H}_{i}, \overline{\mathbf{u}}\right), \mathbf{x}(k) \in \mathfrak{R}_{i}$, 
$i \in I$; according to Lemma 5 and (10), the saturated control input sat $(\mathbf{u}(k))$ can be written in the following form:

$$
\begin{aligned}
& \text { sat }(\mathbf{u}(k)) \\
&=\sum_{s=1}^{2^{m}} \rho_{i s}(k)\left(\mathbf{W}_{i s} \mathbf{K}_{i} \mathbf{C}_{i}+\mathbf{W}_{i s} \Delta \mathbf{K}_{i} \mathbf{C}_{i}+2 \overline{\mathbf{W}_{i s}} \mathbf{H}_{i}\right) \mathbf{x}(k), \\
& \qquad \mathbf{x}(k) \in \mathfrak{R}_{i}, \quad i \in I,
\end{aligned}
$$

where $\rho_{i 1}(K) \geq 0, \rho_{i 2}(K) \geq 0, \ldots, \rho_{i 2^{m}}(K) \geq 0$, and $\sum_{s=1}^{2^{m}} \rho_{i s}(k)=1$.

The closed-loop singular piecewise-affine control system consisting of system (1) and the saturated control input (11) can be described as

$$
\begin{aligned}
& \mathbf{E x}(k+1)= {\left[\overline{\mathbf{A}}_{i}+\mathbf{B}_{i} \widetilde{\mathbf{W}_{i s}}(k)\right] \mathbf{x}(k) } \\
&+\mathbf{D}_{i 1} \mathbf{w}(k)+\mathbf{E}\left(\mathbf{b}_{i}+\Delta \mathbf{b}_{i}\right), \\
& \mathbf{z}(k)=\left[\mathbf{F}_{i}+\mathbf{G}_{i} \widetilde{\mathbf{W}_{i s}}(k)\right] \mathbf{x}(k)+\mathbf{D}_{i 2} \mathbf{w}(k), \\
& \mathbf{x}(k) \in \mathfrak{R}_{i}, \quad i \in I,
\end{aligned}
$$

where $\overline{\mathbf{A}_{i}}=\mathbf{A}_{i}+\Delta \mathbf{A}_{i}, \widetilde{\mathbf{W}_{i s}}(k)=\sum_{s=1}^{2^{m}} \rho_{i s}(k)\left(\mathbf{W}_{i s} \mathbf{K}_{i} \mathbf{C}_{i}+\right.$ $\left.\mathbf{W}_{i s} \Delta \mathbf{K}_{i} \mathbf{C}_{i}+2 \overline{\mathbf{W}_{i s}} \mathbf{H}_{i}\right) \mathbf{x}(k)$.

(B) Resilient Filter for Discrete-Time Singular Piecewise-Affine Systems with Uncertain Parameters. We consider a resilient filtering for discrete-time singular piecewise-affine systems with uncertain parameters as follows:

$$
\begin{gathered}
\widehat{\mathbf{x}}(k+1)=\mathbf{A}_{f} \widehat{\mathbf{x}}(k)+\mathbf{B}_{f} \mathbf{y}(k), \\
\widehat{\mathbf{z}}(k)=\mathbf{C}_{f} \widehat{\mathbf{x}}(k)+\mathbf{D}_{f} \mathbf{y}(k), \quad \mathbf{y}(k) \in \mathfrak{R}_{i}, \quad i \in I,
\end{gathered}
$$

$$
\mathbf{u}(k)=\left(\mathbf{K}_{i}+\Delta \mathbf{K}_{i}\right) \widehat{\mathbf{x}}(k),
$$

where $\mathbf{A}_{f}, \mathbf{B}_{f}, \mathbf{C}_{f}$, and $\mathbf{D}_{f}$ are the design parameters.

According to (11), the saturated control input $\operatorname{sat}(\mathbf{u}(k))$ can be written in the following form:

$$
\begin{array}{r}
\operatorname{sat}(\mathbf{u}(k))=\sum_{s=1}^{2^{m}} \rho_{i s}(k)\left(\mathbf{W}_{i s} \mathbf{K}_{i}+\mathbf{W}_{i s} \Delta \mathbf{K}_{i}+2 \overline{\mathbf{W}_{i s}} \mathbf{H}_{i}\right) \widehat{\mathbf{x}}(k), \\
\widehat{\mathbf{x}}(k) \in \mathfrak{R}_{i}, \quad i \in I .
\end{array}
$$

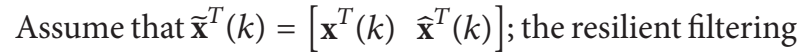
error dynamic system consisting of system (1), 2 , and the saturated control input (14) can be described as

$$
\begin{aligned}
\overline{\mathbf{E}} \widetilde{\mathbf{x}}(k+1) & =\overline{\mathbf{A}} \widetilde{\mathbf{x}}(k)+\overline{\mathbf{B}} \mathbf{w}(k)+\overline{\mathbf{b}}_{i}, \\
\widetilde{\mathbf{z}}(k) & =\overline{\mathbf{C}} \widetilde{\mathbf{x}}(k)+\mathbf{D}_{i 2} \mathbf{w}(k),
\end{aligned}
$$

where $\overline{\mathbf{C}}=\left[F_{i}-\mathbf{D}_{f} \mathbf{C}_{i} \mathbf{G}_{i} \widehat{\mathbf{W}}_{i s}(k)-\mathbf{C}_{f}\right], \overline{\mathbf{A}}=$ $\left[\begin{array}{cc}\mathbf{A}_{i}+\Delta \mathbf{A}_{i} & \mathbf{B}_{i} \widehat{\mathbf{W}}_{i s}(k) \\ \mathbf{B}_{f} \mathbf{C}_{i} & \mathbf{A}_{f}\end{array}\right], \overline{\mathbf{E}}=\left[\begin{array}{cc}\mathbf{E} & \mathbf{0} \\ \mathbf{0} & \mathbf{I}\end{array}\right], \widehat{\mathbf{W}}_{i s}(k)=\sum_{s=1}^{2^{m}} \rho_{i s}(k)\left(\mathbf{W}_{i s} \mathbf{K}_{i}+\right.$ $\left.\mathbf{W}_{i s} \Delta \mathbf{K}_{i}+2 \overline{\mathbf{W}_{i s}} \mathbf{H}_{i}\right) \widehat{\mathbf{x}}(k), \overline{\mathbf{B}}=\left[\begin{array}{c}\mathbf{D}_{i 1} \\ \mathbf{0}\end{array}\right]$, and $\widetilde{\mathbf{z}}(k)=\mathbf{z}(k)-\widehat{\mathbf{z}}(k) \overline{\mathbf{b}_{i}}=$ $\left[\begin{array}{c}\mathbf{E}\left(\mathbf{b}_{i}+\Delta \mathbf{b}_{i}\right) \\ \mathbf{0}\end{array}\right]$.

\section{Main Results}

Theorem 7. If there exist matrices $0<\mathbf{P}_{i}=\mathbf{P}_{i}^{T} \in \mathbf{R}^{n_{x} \times n_{x}}, \mathbf{K}_{i} \in$ $\mathbf{R}^{n_{u} \times n_{y}}, i \in I$, and positive scalar $\beta \leq \beta_{M}, \varepsilon_{i j}, \varepsilon_{i j}^{\prime}, \varepsilon_{i j}^{\prime \prime}, i \in I$, $\lambda_{i j}, i \in I_{1},(i, j) \in \Omega$, such that the following LMIs hold:

$$
\mathbf{E}^{T} \mathbf{P}_{i} \mathbf{E} \geq 0
$$

$$
\left[\begin{array}{ccccccc}
-\varepsilon_{i j}^{\prime \prime} \mathbf{I}_{s 2} & \mathbf{0} & \mathbf{0} & \mathbf{0} & \mathbf{0} & \mathbf{0} & \mathbf{E}_{i 3} \mathbf{C}_{i} \\
* & -\varepsilon_{i j}^{\prime} \mathbf{I}_{s 2} & \mathbf{0} & \mathbf{0} & \mathbf{0} & \mathbf{0} & \mathbf{E}_{i 3} \mathbf{C}_{i} \\
* & * & -\varepsilon_{i j} \mathbf{I}_{s 2} & \mathbf{0} & \mathbf{0} & \mathbf{0} & \mathbf{E}_{i 1} \\
* & * & * & \phi & \mathbf{0} & \mathbf{D}_{i 2} & \Xi \\
* & * & * & * & -\mathbf{P}_{j}^{-1}+\Psi & \mathbf{D}_{i 1} & \Lambda \\
* & * & * & * & * & -\mathbf{I} & \mathbf{0} \\
* & * & * & * & * & * & -\mathbf{E}^{T} \mathbf{P}_{i} \mathbf{E}
\end{array}\right]<0
$$

$$
i \in I_{0}, \quad(i, j) \in \Omega, s \in\left[1,2^{m}\right],
$$

$$
\begin{aligned}
& {\left[\begin{array}{cccccccc}
-\varepsilon_{i j}^{\prime \prime} \mathbf{I}_{s 2} & \mathbf{0} & \mathbf{0} & \mathbf{0} & \mathbf{0} & \mathbf{0} & \mathbf{E}_{i 3} \mathbf{C}_{i} & \mathbf{0} \\
* & -\varepsilon_{i j}^{\prime} \mathbf{I}_{s 2} & \mathbf{0} & \mathbf{0} & \mathbf{0} & \mathbf{0} & \mathbf{E}_{i 3} \mathbf{C}_{i} & \mathbf{0} \\
* & * & -\varepsilon_{i j} \mathbf{I}_{s 2}+\lambda_{i j}^{-1} \mathbf{E}_{i 2} \mathbf{E}_{i 2}^{T} & \mathbf{0} & \lambda_{i j}^{-1} \mathbf{E}_{i 2}\left(\mathbf{E b}_{i}\right)^{T} & \mathbf{0} & \mathbf{E}_{i 1}+\omega & \mathbf{E}_{i 2} f_{i}^{T} \\
* & * & * & \phi & \mathbf{0} & \mathbf{D}_{i 2} & \Xi & \mathbf{0} \\
* & * & * & * & -\mathbf{P}_{j}^{-1}+\Psi+\Phi & \mathbf{D}_{i 1} & \Lambda+\theta & \mathbf{E b}_{i} f_{i}^{T} \\
* & * & * & * & * & -\mathbf{I} & \mathbf{0} & \mathbf{0} \\
* & * & * & * & * & * & \Pi+\vartheta & \lambda_{i j} \overline{\mathbf{F}}_{i}^{T} f_{i} f_{i}^{T} \\
* & * & * & * & * & * & * & \lambda_{i j}\left(f_{i}^{T} f_{i}-I\right)
\end{array}\right]<0} \\
& i \in I_{1}, \quad(i, j) \in \Omega, s \in\left[1,2^{m}\right],
\end{aligned}
$$




$$
\begin{gathered}
{\left[\begin{array}{cc}
1 & \mathbf{Z}_{i l} \\
\mathbf{Z}_{i l}^{T} & \left(\frac{\bar{u}^{2}}{\beta}\right) \mathbf{P}_{i}^{-1}
\end{array}\right] \geq 0, \quad i \in I, \quad l \in[1, m],} \\
{\left[\begin{array}{cc}
1 & \mathbf{L}_{k} \mathbf{P}_{i}^{-1} \\
\mathbf{P}_{i}^{-1} \mathbf{L}_{k}^{T} & \left(\frac{\overline{\mathbf{g}}_{k}^{2}}{\beta}\right) \mathbf{P}_{i}^{-1}
\end{array}\right] \geq 0, \quad i \in I, k \in[1, r],}
\end{gathered}
$$

where $\overline{\mathbf{Q}_{i s}}=\mathbf{Q}_{i s} \mathbf{P}_{i}^{-1}=\left(\mathbf{W}_{i s} \mathbf{K}_{i} \mathbf{C}_{i}+\overline{\mathbf{W}_{i s}} \mathbf{H}_{i}\right) \mathbf{P}_{i}^{-1}=\mathbf{W}_{i s} \mathbf{Y}_{i}+\overline{\mathbf{W}_{i s}} \mathbf{Z}_{i}$, $\mathbf{Z}_{i l}$ is the lth row of matrix $\mathbf{Z}_{i}, \mathbf{L}_{k}$ is the kth row of matrix $\mathbf{L}$, and $\overline{\mathbf{g}_{k}}$ is the kth row of vector $\overline{\mathbf{g}}$, then, for any initial condition $\mathbf{x}_{0}$ starting from the region $\cup_{i \in I}\left(\varepsilon\left(\mathbf{P}_{i}, \beta\right) \cap \mathfrak{R}_{i}\right)$, the discretetime singular piecewise-affine system (1) can be asymptotically stabilized by the controller (10) with $\mathbf{K}_{i}=\mathbf{Y}_{i} \mathbf{P}_{i} \mathbf{C}_{i}^{-1}$. Consider

$$
\begin{gathered}
\boldsymbol{\alpha}=\mathbf{G}_{i} \mathbf{W}_{i s} \mathbf{W}_{i 1}, \quad \boldsymbol{\varphi}=\mathbf{B}_{i} \mathbf{W}_{i s} \mathbf{W}_{i 1}, \\
\Xi=\mathbf{F}_{i}+\mathbf{G}_{i} \mathbf{Q}_{i s}+\mathbf{G}_{i} \overline{\mathbf{W}_{i s}} \mathbf{H}_{i}, \quad \phi=-\gamma^{2} \mathbf{I}+\varepsilon_{i j}^{\prime} \boldsymbol{\alpha} \boldsymbol{\alpha}^{T}, \\
\Lambda=\mathbf{A}_{i}+\mathbf{B}_{i} \mathbf{Q}_{i s}+\mathbf{B}_{i} \overline{\mathbf{W}_{i s}} \mathbf{H}_{i}, \quad \eta=\lambda_{i j} \overline{\mathbf{F}}_{i}^{T} f_{i} f_{i}^{T}, \\
\iota=\lambda_{i j}\left(f_{i}^{T} f_{i}-I\right), \quad \Phi=\lambda_{i j}^{-1} \mathbf{E}_{i}\left(\mathbf{E} \mathbf{b}_{i}\right)^{T}, \\
\Theta=\mathbf{E}_{i 2}\left(\overline{\mathbf{F}}_{i}^{T} f_{i}\right)^{T}, \quad \theta=\mathbf{E b}_{i}\left(\overline{\mathbf{F}}_{i}^{T} f_{i}\right)^{T}, \\
\vartheta=\lambda_{i j} \overline{\mathbf{F}}_{i}^{T} f_{i}\left(\overline{\mathbf{F}}_{i}^{T} f_{i}\right)^{T}, \quad \Psi=\varepsilon_{i j} \mathbf{W}_{i 1} \mathbf{W}_{i 1}^{T}+\varepsilon_{i j}^{\prime \prime} \boldsymbol{\varphi} \boldsymbol{\varphi}^{T} .
\end{gathered}
$$

Proof. In this paper, we consider the following singular piecewise quadratic Lyapunov function:

$$
\mathbf{V}(k, \mathbf{x}(k))=\mathbf{x}^{T}(k) \mathbf{E}^{T} \mathbf{P}_{i} \mathbf{E x}(k), \quad i \in I .
$$

According to the Lyapunov function defined in (22), to make the closed-loop system (12) possess $\mathrm{H}$-infinity performance $\gamma$, we know that it suffices to show the following inequality:

$$
\begin{aligned}
& \mathbf{V}(k+1, \mathbf{x}(k+1))-\mathbf{V}(k, \mathbf{x}(k)) \\
& \quad+\gamma^{-2} \mathbf{z}^{T}(k) \mathbf{z}(k)-\mathbf{w}^{T}(k) \mathbf{w}(k)<0 .
\end{aligned}
$$

In the case of $i \in I_{1},(i, j) \in \Omega$, it follows from (23) that

$$
\begin{aligned}
& \left\{\left[\overline{\mathbf{A}}_{i}+\mathbf{B}_{i} \widetilde{\mathbf{W}_{i s}}(k)\right] \mathbf{x}(k)+\mathbf{D}_{i 1} \mathbf{w}(k)+\mathbf{E}\left(\mathbf{b}_{i}+\Delta \mathbf{b}_{i}\right)\right\}^{T} \\
& \quad \times \mathbf{P}_{j}\left\{\left[\overline{\mathbf{A}}_{i}+\mathbf{B}_{i} \widetilde{\mathbf{W}_{i s}}(k)\right] \mathbf{x}(k)+\mathbf{D}_{i 1} \mathbf{w}(k)+\mathbf{E}\left(\mathbf{b}_{i}+\Delta \mathbf{b}_{i}\right)\right\} \\
& \quad-\mathbf{x}(k)^{T} \mathbf{E}^{T} \mathbf{P}_{i} \mathbf{E} \mathbf{x}(k) \\
& \quad+\gamma^{-2}\left\{\left[\mathbf{F}_{i}+\mathbf{G}_{i} \widetilde{\mathbf{W}_{i s}}(k)\right] \mathbf{x}(k)+\mathbf{D}_{i 2} \mathbf{w}(k)\right\}^{T} \\
& \quad \times\left\{\left[\mathbf{F}_{i}+\mathbf{G}_{i} \widetilde{\mathbf{W}_{i s}}(k)\right] \mathbf{x}(k)+\mathbf{D}_{i 2} \mathbf{w}(k)\right\}-\mathbf{w}(k)^{T} \mathbf{w}(k)<0 .
\end{aligned}
$$

Equation (24) can be rewritten as follows with $(i, j) \in \Omega$ for any nonzero $\mathbf{w}(k) \in \mathbf{l}_{2}[0, \infty)$ :

$$
\begin{aligned}
{\left[\begin{array}{c}
\mathbf{w}(k) \\
\mathbf{x}(k) \\
1
\end{array}\right]^{T}\left\{\left[\begin{array}{c}
\mathbf{D}_{i 1}^{T} \\
\overline{\mathbf{A}}^{T} \\
\left(\mathbf{E}\left(\mathbf{b}_{i}+\Delta \mathbf{b}_{i}\right)\right)^{T}
\end{array}\right] \mathbf{P}_{j}\left(^{*}\right)\right.} \\
+\gamma^{-2}\left[\begin{array}{c}
\mathbf{D}_{i 2}^{T} \\
\overline{\mathbf{B}}^{T} \\
\mathbf{0}
\end{array}\right]\left({ }^{*}\right) \\
\left.+\left[\begin{array}{ccc}
-\mathbf{I} & \mathbf{0} & \mathbf{0} \\
* & -\mathbf{E}^{T} \mathbf{P}_{i} \mathbf{E} & \mathbf{0} \\
* & * & \mathbf{0}
\end{array}\right]\right\}
\end{aligned}
$$

$$
\times\left[\begin{array}{c}
\mathbf{w}(k) \\
\mathbf{x}(k) \\
1
\end{array}\right]<0,
$$

where $\overline{\mathbf{A}}=\overline{\mathbf{A}_{i}}+\mathbf{B}_{i} \widetilde{\mathbf{W}_{i s}}(k), \overline{\mathbf{B}}=\mathbf{F}_{i}+\mathbf{G}_{i} \widetilde{\mathbf{W}_{i s}}(k)$.

From (25), we get

$$
\overline{\mathbf{A}}^{T} \mathbf{P}_{j} \overline{\mathbf{A}}-\mathbf{E}^{T} \mathbf{P}_{i} \mathbf{E}+\gamma^{-2} \overline{\mathbf{B}}^{T} \overline{\mathbf{B}}<0
$$

It is easy to see the following inequality:

$$
\overline{\mathbf{A}}^{T} \mathbf{P}_{j} \overline{\mathbf{A}}-\mathbf{E}^{T} \mathbf{P}_{i} \mathbf{E}<0 .
$$

Assume that $(\mathbf{E}, \overline{\mathbf{A}})$ is not causal We multiply (27) by the grade 1 eigenvectors $\nu^{1}$ and its Hermitian $\nu^{1^{*}}$, respectively. In view of Definition 3, replacing $\overline{\mathbf{A}} \boldsymbol{\nu}^{1}$ by $\mathbf{E} \boldsymbol{\nu}^{2}$ and noting that $\mathrm{E} \boldsymbol{v}^{1}=0$, it gives

$$
\boldsymbol{v}^{2 *} \mathbf{E}^{T} \mathbf{P}_{i} \mathbf{E} \boldsymbol{\nu}^{2}<0
$$

which contradicts (16). Therefore $(\mathbf{E}, \overline{\mathbf{A}})$ is causal. Thus the regularity of $(\mathbf{E}, \overline{\mathbf{A}})$ is implied. As a result, the closed-loop system (12) is said to be admissible.

Then, by taking into consideration the partition information (9) and applying the S-procedure, we have that the 
following inequality implies (25) with $\lambda_{i j}<0, i \in I_{1},(i, j) \in$ $\Omega$ :

$$
\begin{gathered}
{\left[\begin{array}{c}
\mathbf{w}(t) \\
\mathbf{x}(t) \\
1
\end{array}\right]^{T}\left\{\left[\begin{array}{c}
\mathbf{D}_{i 1}^{T} \\
\overline{\mathbf{A}}^{T} \\
\left(\mathbf{E}\left(\mathbf{b}_{i}+\Delta \mathbf{b}_{i}\right)\right)^{T}
\end{array}\right] \mathbf{P}_{j}\left(^{*}\right)\right.} \\
+\gamma^{-2}\left[\begin{array}{c}
\mathbf{D}_{i 2}^{T} \\
\overline{\mathbf{B}}^{T} \\
\mathbf{0}
\end{array}\right]\left({ }^{*}\right) \\
+\left[\begin{array}{ccc}
-\mathbf{I} & \mathbf{0} & \mathbf{0} \\
* & -\mathbf{E}^{T} \mathbf{P}_{i} \mathbf{E} & \mathbf{0} \\
* & * & \mathbf{0}
\end{array}\right]
\end{gathered}
$$

$$
\left.+\lambda_{i j}\left[\begin{array}{ccc}
\mathbf{0} & \mathbf{0} & \mathbf{0} \\
* & \overline{\mathbf{F}}_{i}^{T} \overline{\mathbf{F}}_{i} & \overline{\mathbf{F}}_{i}^{T} f_{i} \\
* & * & f_{i}^{T} f_{i}-1
\end{array}\right]\right\}
$$$$
\times\left[\begin{array}{c}
\mathbf{w}(t) \\
\mathbf{x}(t) \\
1
\end{array}\right]<0, \quad(i, j) \in \Omega
$$

For the matrix inequalities in (30), taking linear combinations over $s$ and using the well-known Schur complement, it is easy to see that the following inequality implies (29):

$$
\begin{gathered}
{\left[\begin{array}{ccccc}
-\gamma^{2} \mathbf{I} & \mathbf{0} & \mathbf{D}_{i 2} & \mathbf{F}_{i}+\mathbf{G}_{i} \mathbf{Q}_{i s}+\mathbf{G}_{i} \overline{\mathbf{W}_{i s}} \mathbf{H}_{i}+\mathbf{G}_{i} \mathbf{W}_{i s} \Delta \mathbf{K}_{i} \mathbf{C}_{i} & \mathbf{0} \\
* & -\mathbf{P}_{j}^{-1} & \mathbf{D}_{i 1} & \mathbf{A}_{i}+\mathbf{B}_{i} \mathbf{Q}_{i s}+\mathbf{B}_{i} \overline{\mathbf{W}_{i s}} \mathbf{H}_{i}+\mathbf{B}_{i} \mathbf{W}_{i s} \Delta \mathbf{K}_{i} \mathbf{C}_{i} & \mathbf{E b}_{i} \\
* & * & -\mathbf{I} & \mathbf{0} & \mathbf{0} \\
* & * & * & -\mathbf{E}^{T} \mathbf{P}_{i} \mathbf{E}+\lambda_{i j} \overline{\mathbf{F}}_{i}^{T} \overline{\mathbf{F}}_{i} & \lambda_{i j} \overline{\mathbf{F}}_{i}^{T} f_{i} \\
* & * & * & * & \lambda_{i j}\left(f_{i}^{T} f_{i}-1\right)
\end{array}\right]} \\
+\left[\begin{array}{ccccc}
\mathbf{0} & \mathbf{0} & \mathbf{0} & \mathbf{0} & \mathbf{0} \\
* & \mathbf{0} & \mathbf{0} & \Delta \mathbf{A}_{i} & \mathbf{E} \Delta \mathbf{b}_{i} \\
* & * & \mathbf{0} & \mathbf{0} & \mathbf{0} \\
* & * & * & \mathbf{0} & \mathbf{0} \\
* & * & * & * & \mathbf{0}
\end{array}\right]<0, \quad i \in I_{1},(i, j) \in \Omega, s \in\left[1,2^{m}\right],
\end{gathered}
$$

where $\mathbf{Q}_{i s}=\mathbf{W}_{i s} \mathbf{K}_{i} \mathbf{C}_{i}+\overline{\mathbf{W}_{i s}} \mathbf{H}_{i}$. On the other hand, by using the relations given in (3), the left-hand side (LHS) of (30) can be easily rewritten as follows:

$$
\begin{aligned}
\operatorname{LHS}(30)= & {\left[\begin{array}{ccccc}
-\gamma^{2} \mathbf{I} & \mathbf{0} & \mathbf{D}_{i 2} & \mathbf{F}_{i}+\mathbf{G}_{i} \mathbf{Q}_{i s}+\mathbf{G}_{i} \overline{\mathbf{W}_{i s}} \mathbf{H}_{i}+\mathbf{G}_{i} \mathbf{W}_{i s} \Delta \mathbf{K}_{i} \mathbf{C}_{i} & \mathbf{0} \\
* & -\mathbf{P}_{j}^{-1} & \mathbf{D}_{i 1} & \mathbf{A}_{i}+\mathbf{B}_{i} \mathbf{Q}_{i s}+\mathbf{B}_{i} \overline{\mathbf{W}_{i s}} \mathbf{H}_{i}+\mathbf{B}_{i} \mathbf{W}_{i s} \Delta \mathbf{K}_{i} \mathbf{C}_{i} & \mathbf{E b}_{i} \\
* & * & -\mathbf{I} & \mathbf{0} & \mathbf{0} \\
* & * & * & -\mathbf{E}^{T} \mathbf{P}_{i} \mathbf{E}+\lambda_{i j} \overline{\mathbf{F}}_{i}^{T} \overline{\mathbf{F}}_{i} & \lambda_{i j} \overline{\mathbf{F}}_{i}^{T} f_{i} \\
* & * & * & * & \lambda_{i j}\left(f_{i}^{T} f_{i}-1\right)
\end{array}\right] } \\
& +\operatorname{Sym}\left\{\left[\begin{array}{c}
\mathbf{0} \\
\mathbf{W}_{i 1} \\
\mathbf{0} \\
\mathbf{0} \\
\mathbf{0}
\end{array}\right] \Delta_{i}(t)\left[\begin{array}{lllll}
\mathbf{0} & \mathbf{0} & \mathbf{0} & \mathbf{E}_{i 1} & \mathbf{E}_{i 2}
\end{array}\right]\right\} .
\end{aligned}
$$


Remark 8. Firstly, we consider the uncertainty terms appearing in the matrices $\mathbf{A}_{i}$ and $\mathbf{b}_{i}$; according to the relations $\Delta \mathbf{K}_{i}=\mathbf{W}_{i 1} \Delta_{i}(t) \mathbf{E}_{i 3}, i \in I$, we can eliminate the uncertainty terms appearing in $\mathbf{G}_{i} \mathbf{W}_{i s} \Delta \mathbf{K}_{i} \mathbf{C}_{i}$ and $\mathbf{B}_{i} \mathbf{W}_{i s} \Delta \mathbf{K}_{i} \mathbf{C}_{i}$ by the same means. $\operatorname{Sym}\{\boldsymbol{\Gamma}\}$ is the shorthand notation for $\Gamma+\Gamma^{T}$.

Thus, based on Lemma 6, by introducing a set of positive scalar parameters $\varepsilon_{i j}>0, i \in I_{1},(i, j) \in \Omega$, it is easy to see that the following inequality implies (31):

$$
\left[\begin{array}{cccccc}
-\varepsilon_{i j} \mathbf{I}_{s 2} & \mathbf{0} & \mathbf{0} & \mathbf{0} & \mathbf{E}_{i 1} & \mathbf{E}_{i 2} \\
* & -\gamma^{2} \mathbf{I} & \mathbf{0} & \mathbf{D}_{i 2} & \mathbf{F}_{i}+\mathbf{G}_{i} \mathbf{Q}_{i s}+\mathbf{G}_{i} \overline{\mathbf{W}_{i s}} \mathbf{H}_{i}+\mathbf{G}_{i} \mathbf{W}_{i s} \Delta \mathbf{K}_{i} \mathbf{C}_{i} & \mathbf{0} \\
* & * & -\mathbf{P}_{j}^{-1}+\varepsilon_{i j} \mathbf{W}_{i 1} \mathbf{W}_{i 1}^{T} & \mathbf{D}_{i 1} & \mathbf{A}_{i}+\mathbf{B}_{i} \mathbf{Q}_{i s}+\mathbf{B}_{i} \overline{\mathbf{W}_{i s}} \mathbf{H}_{i}+\mathbf{B}_{i} \mathbf{W}_{i s} \Delta \mathbf{K}_{i} \mathbf{C}_{i} & \mathbf{E b}_{i} \\
* & * & * & -\mathbf{I} & \mathbf{0} & \mathbf{0} \\
* & * & * & * & -\mathbf{E}^{T} \mathbf{P}_{i} \mathbf{E}+\lambda_{i j} \overline{\mathbf{F}}_{i}^{T} \overline{\mathbf{F}}_{i} & \lambda_{i j} \overline{\mathbf{F}}_{i}^{T} f_{i} \\
* & * & * & * & * & \lambda_{i j}\left(f_{i}^{T} f_{i}-1\right)
\end{array}\right]<0 .
$$

For the matrix inequalities in (33), introduce two sets of positive scalar parameters $\varepsilon_{i j}^{\prime}, \varepsilon_{i j}^{\prime \prime}$ :

$$
\left[\begin{array}{cccccccc}
-\varepsilon_{i j}^{\prime \prime} \mathbf{I}_{s 2} & \mathbf{0} & \mathbf{0} & \mathbf{0} & \mathbf{0} & \mathbf{0} & \mathbf{E}_{i 3} \mathbf{C}_{i} & \mathbf{0} \\
* & -\varepsilon_{i j}^{\prime} \mathbf{I}_{s 2} & \mathbf{0} & \mathbf{0} & \mathbf{0} & \mathbf{0} & \mathbf{E}_{i 3} \mathbf{C}_{i} & \mathbf{0} \\
* & * & -\varepsilon_{i j} \mathbf{I}_{s 2} & \mathbf{0} & \mathbf{0} & \mathbf{0} & \mathbf{E}_{i 1} & \mathbf{E}_{i 2} \\
* & * & * & -\gamma^{2} \mathbf{I}+\varepsilon_{i j}^{\prime} \boldsymbol{\alpha} \boldsymbol{\alpha}^{T} & \mathbf{0} & \mathbf{D}_{i 2} & \Xi & \mathbf{0} \\
* & * & * & * & -\mathbf{P}_{j}^{-1}+\Psi & \mathbf{D}_{i 1} & \Lambda & \mathbf{E b}_{i} \\
* & * & * & * & * & -\mathbf{I} & \mathbf{0} & \mathbf{0} \\
* & * & * & * & * & * & \Pi & \lambda_{i j} \overline{\mathbf{F}}_{i}^{T} f_{i} \\
* & * & * & * & * & * & * & \lambda_{i j}\left(f_{i}^{T} f_{i}-1\right)
\end{array}\right]<0
$$

where $\boldsymbol{\alpha}=\mathbf{G}_{i} \mathbf{W}_{i s} \mathbf{W}_{i 1}, \boldsymbol{\varphi}=\mathbf{B}_{i} \mathbf{W}_{i s} \mathbf{W}_{i 1}, \Xi=\mathbf{F}_{i}+\mathbf{G}_{i} \mathbf{Q}_{i s}+$ $\mathbf{G}_{i} \overline{\mathbf{W}_{i s}} \mathbf{H}_{i}, \Lambda=\mathbf{A}_{i}+\mathbf{B}_{i} \mathbf{Q}_{i s}+\mathbf{B}_{i} \overline{\mathbf{W}_{i s}} \mathbf{H}_{i}, \Pi=-\mathbf{E}^{T} \mathbf{P}_{i} \mathbf{E}+\lambda_{i j} \overline{\mathbf{F}}_{i}^{T} \overline{\mathbf{F}}_{i}$, and $\Psi=\varepsilon_{i j} \mathbf{W}_{i 1} \mathbf{W}_{i 1}^{T}+\varepsilon_{i j}^{\prime \prime} \varphi \varphi^{T}$.
Using Schur complement again, it is seen that the above inequality is equivalent to the following inequality:

$$
\begin{aligned}
& {\left[\begin{array}{ccccccc}
-\varepsilon_{i j}^{\prime \prime} \mathbf{I}_{s 2} & \mathbf{0} & \mathbf{0} & \mathbf{0} & \mathbf{0} & \mathbf{0} & \mathbf{E}_{i 3} \mathbf{C}_{i} \\
* & -\varepsilon_{i j}^{\prime} \mathbf{I}_{s 2} & \mathbf{0} & \mathbf{0} & \mathbf{0} & \mathbf{0} & \mathbf{E}_{i 3} \mathbf{C}_{i} \\
* & * & -\varepsilon_{i j} \mathbf{I}_{s 2} & \mathbf{0} & \mathbf{0} & \mathbf{0} & \mathbf{E}_{i 1} \\
* & * & * & -\gamma^{2} \mathbf{I}+\varepsilon_{i j}^{\prime} \boldsymbol{\alpha} \boldsymbol{\alpha}^{T} & \mathbf{0} & \mathbf{D}_{i 2} & \Xi \\
* & * & * & * & -\mathbf{P}_{j}^{-1}+\Psi & \mathbf{D}_{i 1} & \Lambda \\
* & * & * & * & * & -\mathbf{I} & \mathbf{0} \\
* & * & * & * & * & * & \Pi
\end{array}\right]} \\
& +\left(-\lambda_{i j}^{-1}\right)\left[\begin{array}{c}
\mathbf{0} \\
\mathbf{0} \\
\mathbf{E}_{i 2} \\
\mathbf{0} \\
\mathbf{E b}_{i} \\
\mathbf{0} \\
\lambda_{i j} \overline{\mathbf{F}}_{i}^{T} f_{i}
\end{array}\right]\left(f_{i}^{T} f_{i}-1\right)^{-1}\left[\begin{array}{c}
\mathbf{0} \\
\mathbf{0} \\
\mathbf{E}_{i 2} \\
\mathbf{0} \\
\mathbf{E} \mathbf{b}_{i} \\
\mathbf{0} \\
\lambda_{i j} \overline{\mathbf{F}}_{i}^{T} f_{i}
\end{array}\right]^{T}<0 .
\end{aligned}
$$

By the well-known fact $\left(f_{i}^{T} f_{i}-1\right)^{-1}=-1+f_{i}^{T} \times$ $\left(f_{i} f_{i}^{T}-I\right)^{-1} f_{i}$ (matrix inversion lemma) and some simple calculations, the matrix inequality (34) can be rewritten as follows: 


$$
\begin{aligned}
& {\left[\begin{array}{ccccccc}
-\varepsilon_{i j}^{\prime \prime} \mathbf{I}_{s 2} & \mathbf{0} & \mathbf{0} & \mathbf{0} & \mathbf{0} & \mathbf{0} & \mathbf{E}_{i 3} \mathbf{C}_{i} \\
* & -\varepsilon_{i j}^{\prime} \mathbf{I}_{s 2} & \mathbf{0} & \mathbf{0} & \mathbf{0} & \mathbf{0} & \mathbf{E}_{i 3} \mathbf{C}_{i} \\
* & * & -\varepsilon_{i j} \mathbf{I}_{s 2}+\lambda_{i j}^{-1} \mathbf{E}_{i 2} \mathbf{E}_{i 2}^{T} & \mathbf{0} & \lambda_{i j}^{-1} \mathbf{E}_{i 2}\left(\mathbf{E b}_{i}\right)^{T} & \mathbf{0} & \mathbf{E}_{i 1}+\emptyset \\
* & * & * & -\gamma^{2} \mathbf{I}+\varepsilon_{i j}^{\prime} \boldsymbol{\alpha} \boldsymbol{\alpha}^{T} & \mathbf{0} & \mathbf{D}_{i 2} & \Xi \\
* & * & * & * & -\mathbf{P}_{j}^{-1}+\Psi+\Phi & \mathbf{D}_{i 1} & \Lambda+\theta \\
* & * & * & * & * & -\mathbf{I} & \mathbf{0} \\
* & * & * & * & * & * & \Pi+\vartheta
\end{array}\right]} \\
& +\left(-\lambda_{i j}^{-1}\right)\left[\begin{array}{c}
\mathbf{0} \\
\mathbf{0} \\
\mathbf{E}_{i 2} f_{i}^{T} \\
\mathbf{0} \\
\mathbf{E b}_{i} f_{i}^{T} \\
\mathbf{0} \\
\lambda_{i j} \overline{\mathbf{F}}_{i}^{T} f_{i} f_{i}^{T}
\end{array}\right]\left(f_{i}^{T} f_{i}-I\right)^{-1}\left[\begin{array}{c}
\mathbf{0} \\
\mathbf{0} \\
\mathbf{E}_{i 2} f_{i}^{T} \\
\mathbf{0} \\
\mathbf{E} \mathbf{b}_{i} f_{i}^{T} \\
\mathbf{0} \\
\lambda_{i j} \overline{\mathbf{F}}_{i}^{T} f_{i} f_{i}^{T}
\end{array}\right]^{T}<0
\end{aligned}
$$

where $\Phi=\lambda_{i j}^{-1} \mathbf{E b}_{i}\left(\mathbf{E b}_{i}\right)^{T}, \boldsymbol{\omega}=\mathbf{E}_{i 2}\left(\overline{\mathbf{F}}_{i}^{T} f_{i}\right)^{T}, \theta=\mathbf{E b}_{i}\left(\overline{\mathbf{F}}_{i}^{T} f_{i}\right)^{T}$, and $\vartheta=\lambda_{i j} \overline{\mathbf{F}}_{i}^{T} f_{i}\left(\overline{\mathbf{F}}_{i}^{T} f_{i}\right)^{T}$.
By using the well-known Schur complement again, it is easy to see that the following inequality implies (35):

$$
\left[\begin{array}{cccccccc}
-\varepsilon_{i j}^{\prime \prime} \mathbf{I}_{s 2} & \mathbf{0} & \mathbf{0} & \mathbf{0} & \mathbf{0} & \mathbf{0} & \mathbf{E}_{i 3} \mathbf{C}_{i} & \mathbf{0} \\
* & -\varepsilon_{i j}^{\prime} \mathbf{I}_{s 2} & \mathbf{0} & \mathbf{0} & \mathbf{0} & \mathbf{0} & \mathbf{E}_{i 3} \mathbf{C}_{i} & \mathbf{0} \\
* & * & -\varepsilon_{i j} \mathbf{I}_{s 2}+\lambda_{i j}^{-1} \mathbf{E}_{i 2} \mathbf{E}_{i 2}^{T} & \mathbf{0} & \lambda_{i j}^{-1} \mathbf{E}_{i 2}\left(\mathbf{E b}_{i}\right)^{T} & \mathbf{0} & \mathbf{E}_{i 1}+\omega & \mathbf{E}_{i 2} f_{i}^{T} \\
* & * & * & \phi & \mathbf{0} & \mathbf{D}_{i 2} & \Xi & \mathbf{0} \\
* & * & * & * & -\mathbf{P}_{j}^{-1}+\Psi+\Phi & \mathbf{D}_{i 1} & \Lambda+\theta & \mathbf{E b}_{i} f_{i}^{T} \\
* & * & * & * & * & -\mathbf{I} & \mathbf{0} & \mathbf{0} \\
* & * & * & * & * & * & \Pi+\vartheta & \lambda_{i j} \overline{\mathbf{F}}_{i}^{T} f_{i} f_{i}^{T} \\
* & * & * & * & * & * & * & \lambda_{i j}\left(f_{i}^{T} f_{i}-I\right)
\end{array}\right]<0
$$

where $\phi=-\gamma^{2} \mathbf{I}+\varepsilon_{i j}^{\prime} \boldsymbol{\alpha} \boldsymbol{\alpha}^{T}$; on the other hand, it follows that $\mathbf{b}_{i}+\Delta \mathbf{b}_{i}=0$; it is easy to see the following inequality:

$$
\begin{aligned}
& \left\{\left[\overline{\mathbf{A}}_{i}+\mathbf{B}_{i} \widetilde{\mathbf{W}_{i s}}(k)\right] \mathbf{x}(k)+\mathbf{D}_{i 1} \mathbf{w}(k)\right\}^{T} \\
& \quad \times \mathbf{P}_{j}\left\{\left[\overline{\mathbf{A}_{i}}+\mathbf{B}_{i} \widetilde{\mathbf{W}_{i s}}(k)\right] \mathbf{x}(k)+\mathbf{D}_{i 1} \mathbf{w}(k)\right\} \\
& \quad-\mathbf{x}(k)^{T} \mathbf{E}^{T} \mathbf{P}_{i} \mathbf{E x}(k) \\
& \quad+\gamma^{-2}\left\{\left[\mathbf{F}_{i}+\mathbf{G}_{i} \widetilde{\mathbf{W}_{i s}}(k)\right] \mathbf{x}(k)+\mathbf{D}_{i 2} \mathbf{w}(k)\right\}^{T} \\
& \quad \times\left\{\left[\mathbf{F}_{i}+\mathbf{G}_{i} \widetilde{\mathbf{W}_{i s}}(k)\right] \mathbf{x}(k)+\mathbf{D}_{i 2} \mathbf{w}(k)\right\} \\
& \quad-\mathbf{w}(k)^{T} \mathbf{w}(k)<0 \quad i \in I_{0}, \quad(i, j) \in \Omega .
\end{aligned}
$$

For the matrix inequalities in (38), taking linear combinations over $s$ and using the Schur complement, one can obtain from (37) that the inequality also holds for $i \in I_{0},(i, j) \in \Omega$. Consider

$$
\begin{gathered}
{\left[\begin{array}{cccc}
-\gamma^{2} \mathbf{I} & \mathbf{0} & \mathbf{D}_{i 2} & \mathbf{F}_{i}+\mathbf{G}_{i} \mathbf{Q}_{i s}+\mathbf{G}_{i} \overline{\mathbf{W}_{i s}} \mathbf{H}_{i}+\mathbf{G}_{i} \mathbf{W}_{i s} \Delta \mathbf{K}_{i} \mathbf{C}_{i} \\
* & -\mathbf{P}_{j}^{-1} & \mathbf{D}_{i 1} & \overline{\mathbf{A}_{i}}+\mathbf{B}_{i} \mathbf{Q}_{i s}+\mathbf{B}_{i} \overline{\mathbf{W}_{i s}} \mathbf{H}_{i}+\mathbf{B}_{i} \mathbf{W}_{i s} \Delta \mathbf{K}_{i} \mathbf{C}_{i} \\
* & * & -\mathbf{I} & \mathbf{0} \\
* & * & * & -\mathbf{E}^{T} \mathbf{P}_{i} \mathbf{E}
\end{array}\right]} \\
\quad<0 .
\end{gathered}
$$

By a similar technique dealing with the uncertainties as in (30), it is easy to see that the matrix inequalities in (38) hold if and only if there exist three sets of positive scalars 
$\varepsilon_{i j}, \varepsilon_{i j}^{\prime}$, and $\varepsilon_{i j}^{\prime \prime}$ such that the matrix inequalities in (39) hold. Consider

$$
\left[\begin{array}{ccccccc}
-\varepsilon_{i j}^{\prime \prime} \mathbf{I}_{s 2} & \mathbf{0} & \mathbf{0} & \mathbf{0} & \mathbf{0} & \mathbf{0} & \mathbf{E}_{i 3} \mathbf{C}_{i} \\
* & -\varepsilon_{i j}^{\prime} \mathbf{I}_{s 2} & \mathbf{0} & \mathbf{0} & \mathbf{0} & \mathbf{0} & \mathbf{E}_{i 3} \mathbf{C}_{i} \\
* & * & -\varepsilon_{i j} \mathbf{I}_{s 2} & \mathbf{0} & \mathbf{0} & \mathbf{0} & \mathbf{E}_{i 1} \\
* & * & * & \phi & \mathbf{0} & \mathbf{D}_{i 2} & \Xi \\
* & * & * & * & -\mathbf{P}_{j}^{-1}+\Psi & \mathbf{D}_{i 1} & \Lambda \\
* & * & * & * & * & -\mathbf{I} & \mathbf{0} \\
* & * & * & * & * & * & -\mathbf{E}^{T} \mathbf{P}_{i} \mathbf{E}
\end{array}\right]<0 .
$$

It is easy to see that the inequalities in (39) and (36) are equivalent to LMIs in (17) and (18).

Remark 9. In the case of $\mathbf{b}_{i}+\Delta \mathbf{b}_{i}=0, i \in I_{0}$, where $I_{0}$ denotes the index set of regions with $f_{i}^{T} f_{i}-1 \leq 0$, the partition information (9) is not considered in the process of Theorem 7 proof. To make the results simple, in Theorem 7 , we only investigate the system matrix $\mathbf{A}_{i}$ and offset term $\mathbf{E b}_{i}$ is time-variant. In fact, when matrices $\mathbf{B}_{i}, \mathbf{D}_{i 1}, \mathbf{D}_{i 2}, \mathbf{F}_{i}$, and $\mathbf{G}_{i}$ are time-variant, we can get the resilient $\mathrm{H}$-infinity output feedback controllers with the same course.

In addition, by Schur complement, the LMIs in (19) are equivalent to $\mathbf{Z}_{i l} \mathbf{X}_{i}^{-1} \mathbf{Z}_{i l}^{T} \leq \bar{u}^{2} / \beta, i \in I, l \in[1, m]$, which are equivalent to $\mathbf{H}_{i l} \mathbf{P}_{i}^{-1} \mathbf{H}_{i l}^{T} \leq \bar{u}^{2} / \beta, i \in I, l \in[1, m]$. It is easy to see that $\mathbf{H}_{i l} \mathbf{P}_{i}^{-1} \mathbf{H}_{i l}^{T} \leq \bar{u}^{2} / \beta, i \in I, l \in[1, m]$ are equivalent to $\varepsilon\left(\mathbf{P}_{i}, \beta\right) \subset \zeta\left(\mathbf{H}_{i}, \bar{u}\right), i \in I$ (see [41]). Similarly, the LMIs in (20) are equivalent to $\varepsilon\left(\mathbf{P}_{i}, \beta\right) \subset \zeta\left(\mathbf{L}_{k}, \overline{\mathbf{g}_{k}}\right), i \in I$, $k \in[1, r]$.
Using inequalities (23) and noticing that $\Delta \mathbf{V}(k)=\mathbf{V}(k+$ $1, \mathbf{x}(k+1))-\mathbf{V}(k, \mathbf{x}(k))$ and $\mathbf{V}(0)=0$, one can obtain

$$
\begin{aligned}
& \mathbf{V}(k, \mathbf{x}(k))= \mathbf{x}^{T}(k) \mathbf{E}^{T} \mathbf{P}_{i} \mathbf{E x}(k) \\
&=\sum_{k=0}^{t-1} \Delta \mathbf{V}(k) \leq \sum_{k=0}^{t-1} \mathbf{w}^{T}(t) \mathbf{w}(t) \leq \beta, \\
& \mathbf{x}(k) \in \mathfrak{R}_{i}, \quad i \in I, \quad t \geq 1 .
\end{aligned}
$$

At the time $k$, it is seen from (40) that $\mathbf{x}(k) \in \varepsilon\left(\mathbf{P}_{i}, \beta\right) \cap$ $\mathfrak{R}_{i}, \mathbf{x}(k) \in \mathfrak{R}_{i}, i \in I$. Using the conditions $\varepsilon\left(\mathbf{P}_{i}, \beta\right) \subset$ $\zeta\left(\mathbf{H}_{i}, \bar{u}\right), i \in I$, and $\varepsilon\left(\mathbf{P}_{i}, \beta\right) \subset \zeta\left(\mathbf{L}_{k}, \overline{\mathbf{g}_{k}}\right), i \in I, k \in[1, r]$, we can obtain the relationships $\mathbf{x}(k) \in \zeta\left(\mathbf{H}_{i}, \bar{u}\right)$ and $-\overline{\mathbf{g}} \leq$ $\mathbf{L x}(k) \leq \overline{\mathbf{g}}$. Assume that, at the next time $k+1$, the state of the closed-loop system (12) transits to $\mathbf{x}(k+1) \in \mathfrak{R}_{j}, j \in I$. It is clear from (40) that $\mathbf{x}(k+1) \in \varepsilon\left(\mathbf{P}_{j}, \beta\right) \cap \Re_{j}$. Then it follows from the conditions $\varepsilon\left(\mathbf{P}_{i}, \beta\right) \subset \zeta\left(\mathbf{H}_{i}, \bar{u}\right), i \in I$, and $\varepsilon\left(\mathbf{P}_{i}, \beta\right) \subset$ $\zeta\left(\mathbf{L}_{k}, \overline{\mathbf{g}_{k}}\right), i \in I, k \in[1, r]$, that $\mathbf{x}(k+1) \in \zeta\left(\mathbf{H}_{j}, \bar{u}\right)$ and $-\overline{\mathbf{g}} \leq \mathbf{L x}(k+1) \leq \overline{\mathbf{g}}$. Using the above deductions recursively, it can be concluded that $\mathbf{x}(k) \in \zeta\left(\mathbf{H}_{i}, \bar{u}\right)$ and $-\overline{\mathbf{g}} \leq \mathbf{L x}(k) \leq \overline{\mathbf{g}}$ always hold for $\mathbf{x}(k) \in \mathfrak{R}_{i}, i \in I, t \geq 0$, and the state trajectory of the closed-loop system (12) will remain in the region $\cup_{i \in I}\left(\varepsilon\left(\mathbf{P}_{i}, \beta\right) \cap \mathfrak{R}_{i}\right)$. This completes the proof.

For a fixed scalar $\beta \leq \beta_{M}$, the smallest $\mathrm{H}$-infinity performance $\gamma$ can be measured by solving this optimization problem: $\left(\min / \mathbf{P}_{i}^{-1}, \mathbf{Y}_{i}, \mathbf{Z}_{i}\right) \gamma$, so that the LMIs in (17)-(20) hold.

Theorem 10. For given positive scalars $\gamma$ and $\beta \leq \beta_{M}$, if there exist matrices $0<\mathbf{H}_{1}=\mathbf{H}_{1}^{T} \in \mathbf{R}^{n_{x} \times n_{x}}, 0<\mathbf{H}_{3}=\mathbf{H}_{3}^{T} \epsilon$ $\mathbf{R}^{n_{x} \times n_{x}}, \mathbf{H}_{2} \in \mathbf{R}^{n_{x} \times n_{x}}, 0<\mathbf{P}_{i}=\mathbf{P}_{i}^{T} \in \mathbf{R}^{2 n_{x} \times 2 n_{x}}, \mathbf{K}_{i} \in \mathbf{R}^{n_{u} \times n_{y}}$, $\mathbf{A}_{f} \in \mathbf{R}^{n_{x} \times n_{x}}, \mathbf{B}_{f} \in \mathbf{R}^{n_{x} \times n_{x}}, \mathbf{C}_{f} \in \mathbf{R}^{n_{x} \times n_{x}}$, and $\mathbf{D}_{f} \in \mathbf{R}^{n_{x} \times n_{x}}$ and positive scalars $\varepsilon_{i j}, \varepsilon_{i j}^{\prime}, \varepsilon_{i j}^{\prime \prime}, i \in I, \lambda_{i j}<0, i \in I_{1},(i, j) \in \Omega$, such that the following LMIs hold:

$$
\mathbf{E}^{T} \mathbf{P}_{i} \mathbf{E} \geq 0
$$

$$
\left[\begin{array}{ccccccccc}
-\varepsilon_{i j}^{\prime \prime} \mathbf{I}_{s 2} & \mathbf{0} & \mathbf{0} & \mathbf{0} & \mathbf{0} & \mathbf{0} & \mathbf{0} & \mathbf{0} & \mathbf{E}_{i 3} \\
* & -\varepsilon_{i j}^{\prime} \mathbf{I}_{s 2} & \mathbf{0} & \mathbf{0} & \mathbf{0} & \mathbf{0} & \mathbf{0} & \mathbf{0} & \mathbf{E}_{i 3} \\
* & * & -\varepsilon_{i j} \mathbf{I}_{s 2} & \mathbf{0} & \mathbf{0} & \mathbf{0} & \mathbf{0} & \mathbf{E}_{i 1} & \mathbf{0} \\
* & * & * & \phi & \mathbf{0} & \mathbf{0} & \mathbf{D}_{i 2} & \mathbf{F}_{i}-\mathbf{D}_{f} \mathbf{C}_{i} & \mathbf{G}_{i} \widehat{\mathbf{Q}}_{i s}+\mathbf{G}_{i} \overline{\mathbf{W}_{i s}} \mathbf{H}_{i}-\mathbf{C}_{f} \\
* & * & * & * & \sigma & \mathbf{H}_{2} & \mathbf{D}_{i 1} & \mathbf{A}_{i} & \mathbf{B}_{i} \widehat{\mathbf{Q}}_{i s}+\mathbf{B}_{i} \overline{\mathbf{W}_{i s}} \mathbf{H}_{i} \\
* & * & * & * & * & \mathbf{H}_{3} & \mathbf{0} & \mathbf{B}_{f} \mathbf{C}_{i} & \mathbf{A}_{f} \\
* & * & * & * & * & * & -\mathbf{I} & \mathbf{0} & \mathbf{0} \\
* & * & * & * & * & * & * & J_{1} & J_{2} \\
* & * & * & * & * & * & * & * & J_{3}
\end{array}\right]<0
$$




$$
\begin{aligned}
& {\left[\begin{array}{cccccccccc}
-\varepsilon_{i j}^{\prime \prime} \mathbf{I}_{s 2} & \mathbf{0} & \mathbf{0} & \mathbf{0} & \mathbf{0} & \mathbf{0} & \mathbf{0} & \mathbf{0} & \mathbf{E}_{i 3} & \mathbf{0} \\
* & -\varepsilon_{i j}^{\prime} \mathbf{I}_{s 2} & \mathbf{0} & \mathbf{0} & \mathbf{0} & \mathbf{0} & \mathbf{0} & \mathbf{0} & \mathbf{E}_{i 3} & \mathbf{0} \\
* & * & -\varepsilon_{i j} \mathbf{I}_{s 2}+\delta & \mathbf{0} & \chi & \mathbf{0} & \mathbf{0} & \mathbf{E}_{i 1}+\lambda_{i j}^{-1} \mathbf{E}_{i 2} \mathbf{I}_{1}^{T} & \lambda_{i j}^{-1} \mathbf{E}_{i 2} \mathbf{I}_{2}^{T} & \mathbf{E}_{i 2} \\
* & * & * & \phi & \mathbf{0} & \mathbf{0} & \mathbf{D}_{i 2} & \mathbf{F}_{i}-\mathbf{D}_{f} \mathbf{C}_{i} & \aleph & \mathbf{0} \\
* & * & * & * & \wp & \mathbf{H}_{2} & \mathbf{D}_{i 1} & \mathbf{A}_{i}+\lambda_{i j}^{-1} \mathbf{E}_{i} \mathbf{I}_{1}^{T} & \mathfrak{\Im} & \mathbf{E b}_{i} \\
* & * & * & * & * & \mathbf{H}_{3} & \mathbf{0} & \mathbf{B}_{f} \mathbf{C}_{i} & \mathbf{A}_{f} & \mathbf{0} \\
* & * & * & * & * & * & -\mathbf{I} & \mathbf{0} & \mathbf{0} & \mathbf{0} \\
* & * & * & * & * & * & * & J_{1}+\lambda_{i j}^{-1} I_{1} I_{1}^{T} & J_{2}+\lambda_{i j}^{-1} I_{1} I_{2}^{T} & I_{1} \\
* & * & * & * & * & * & * & * & J_{3}+\lambda_{i j}^{-1} I_{2} I_{2}^{T} & I_{2} \\
* & * & * & * & * & * & * & * & * & \lambda_{i j}\left(f_{i}^{T} f_{i}-I\right)
\end{array}\right]<0,} \\
& i \in I_{1}, \quad(i, j) \in \Omega, s \in\left[1,2^{m}\right], \\
& {\left[\begin{array}{cc}
1 & \mathbf{Z}_{i l} \\
\mathbf{Z}_{i l}^{T} & \left(\frac{\bar{u}^{2}}{\beta}\right)^{-1} \mathbf{P}_{i}^{-1}
\end{array}\right] \geq 0, \quad i \in I, l \in[1, m],} \\
& {\left[\begin{array}{cc}
1 & \mathbf{L}_{k} \mathbf{P}_{i}^{-1} \\
\mathbf{P}_{i}^{-1} \mathbf{L}_{k}^{T} & \left(\frac{\overline{\mathbf{g}}_{k}^{2}}{\beta}\right)^{-1} \mathbf{P}_{i}^{-1}
\end{array}\right] \geq 0, \quad i \in I, k \in[1, r],}
\end{aligned}
$$

where $\overline{\widehat{\mathbf{Q}}_{i s}}=\widehat{\mathbf{Q}}_{i s} \mathbf{P}_{i}^{-1}=\left(\mathbf{W}_{i s} \mathbf{K}_{i}+\overline{\mathbf{W}_{i s}} \mathbf{H}_{i}\right) \mathbf{P}_{i}^{-1}=\mathbf{W}_{i s} \mathbf{Y}_{i}+\overline{\mathbf{W}_{i s}} \mathbf{Z}_{i}$, $\mathbf{Z}_{i l}$ is the lth row of matrix $\mathbf{Z}_{i}, \mathbf{L}_{k}$ is the kth row of matrix $\mathbf{L}$, and $\overline{\mathbf{g}_{k}}$ is the kth row of vector $\overline{\mathbf{g}}$, then for any initial condition $x_{0}$ starting from the region $\cup_{i \in I}\left(\varepsilon\left(\mathbf{P}_{i}, \beta\right) \cap \mathfrak{R}_{i}\right)$, the discretetime singular piecewise-affine system (1) can be asymptotically stabilized by the resilient $H$-infinity filter 2 with $\mathbf{K}_{i}=\mathbf{Y}_{i} \mathbf{P}_{i}$. Consider

$$
\begin{aligned}
& \wp=\mathbf{H}_{1}+\varepsilon_{i j} \mathbf{W}_{i 1} \mathbf{W}_{i 1}^{T}+\varepsilon_{i j}^{\prime \prime} \varphi \varphi^{T}+\lambda_{i j}^{-1} \mathbf{E b}_{i}\left(\mathbf{E b}_{i}\right)^{T}, \\
& \mathfrak{I}=\mathbf{B}_{i} \widehat{\mathbf{Q}}_{i s}+\mathbf{B}_{i} \overline{\mathbf{W}_{i s}} \mathbf{H}_{i}+\lambda_{i j}^{-1} \mathbf{E b}_{i} I_{2}^{T}, \\
& \aleph=\mathbf{G}_{i} \widehat{\mathbf{Q}}_{i s}+\mathbf{G}_{i} \overline{\mathbf{W}_{i s}} \mathbf{H}_{i}-\mathbf{C}_{f}, \\
& \boldsymbol{\alpha}=\mathbf{G}_{i} \mathbf{W}_{i s} \mathbf{W}_{i 1}, \quad \boldsymbol{\varphi}=\mathbf{B}_{i} \mathbf{W}_{i s} \mathbf{W}_{i 1}, \\
& \phi=-\gamma^{2} \mathbf{I}+\varepsilon_{i j}^{\prime} \boldsymbol{\alpha} \boldsymbol{\alpha}^{T}, \quad \chi=\lambda_{i j}^{-1} \mathbf{E}_{i 2}\left(\mathbf{E b}_{i}\right)^{T}, \\
& \delta=\lambda_{i j}^{-1} \mathbf{E}_{i 2} \mathbf{E}_{i 2}^{T}, \\
& \sigma=\mathbf{H}_{1}+\varepsilon_{i j} \mathbf{W}_{i 1} \mathbf{W}_{i 1}^{T}+\varepsilon_{i j}^{\prime \prime} \boldsymbol{\varphi} \boldsymbol{\varphi}^{T}, \\
& -\mathbf{P}_{j}^{-1}=\left[\begin{array}{cc}
\mathbf{H}_{1} & \mathbf{H}_{2} \\
* & \mathbf{H}_{3}
\end{array}\right], \quad \lambda_{i j} \overline{\mathbf{F}}_{i}^{T} f_{i}=\left[\begin{array}{c}
I_{1} \\
I_{2}
\end{array}\right], \\
& -\overline{\mathbf{E}}^{T} \mathbf{P}_{i} \overline{\mathbf{E}}+\lambda_{i j} \overline{\mathbf{F}}_{i}^{T} \overline{\mathbf{F}}_{i}=\left[\begin{array}{ll}
J_{1} & J_{2} \\
* & J_{3}
\end{array}\right] .
\end{aligned}
$$

Proof. From the proof of Theorem 7, we also consider the singular piecewise quadratic Lyapunov function defined in (22); to make the resilient filtering error dynamic system (15) possess $\mathrm{H}$-infinity performance $\gamma$, we know that it suffices to show the following inequality:

$$
\begin{aligned}
& V(k+1, \widetilde{\mathbf{x}}(k+1))-V(k, \widetilde{\mathbf{x}}(k)) \\
& +\gamma^{-2} \widetilde{\mathbf{z}}^{T}(k) \widetilde{\mathbf{z}}(k)-\mathbf{w}^{T}(k) \mathbf{w}(k)<0,
\end{aligned}
$$

where $\widetilde{\mathbf{z}}(k)=\mathbf{z}(k)-\widehat{\mathbf{z}}(k)$, for any nonzero $\mathbf{w}(k) \in \mathbf{l}_{2}[0, \infty)$, and (44) can be rewritten in the following inequality:

$$
\begin{aligned}
& \left\{\widetilde{\mathbf{x}}(k)^{T} \overline{\mathbf{A}}^{T}+\mathbf{w}(k)^{T} \overline{\mathbf{B}}^{T}+\overline{\mathbf{b}}_{i}^{T}\right\} \\
& \quad \times \mathbf{P}_{j}\left\{\overline{\mathbf{A}} \widetilde{\mathbf{x}}(k)+\overline{\mathbf{B}} \mathbf{w}(k)+\overline{\mathbf{b}_{i}}\right\} \\
& \quad-\widetilde{\mathbf{x}}^{T}(k) \overline{\mathbf{E}}^{T} \mathbf{P}_{i} \overline{\mathbf{E}} \widetilde{\mathbf{x}}(k)-\mathbf{w}(k)^{T} \mathbf{w}(k)+\gamma^{-2} \\
& \quad \times\left\{\widetilde{\mathbf{x}}(k)^{T}\left[\mathbf{F}_{i}-\mathbf{D}_{f} \mathbf{C}_{i} \mathbf{G}_{i} \widehat{\mathbf{W}}_{i s}(k)-\mathbf{C}_{f}\right]^{T}+\mathbf{w}(k)^{T} \mathbf{D}_{i 2}^{T}\right\} \\
& \quad \times\left\{\left[\mathbf{F}_{i}-\mathbf{D}_{f} \mathbf{C}_{i} \quad \mathbf{G}_{i} \widehat{\mathbf{W}}_{i s}(k)-\mathbf{C}_{f}\right] \widetilde{\mathbf{x}}(k)+\mathbf{D}_{i 2} \mathbf{w}(k)\right\}<0 .
\end{aligned}
$$

By the system state-space equation, it is easy to see the following inequality: 


$$
\begin{aligned}
& {\left[\begin{array}{c}
\mathbf{w}(k) \\
\widetilde{\mathbf{x}}(k) \\
1
\end{array}\right]^{T}} \\
& \times\left[\begin{array}{ccc}
\overline{\mathbf{B}}^{T} \mathbf{P}_{j} \overline{\mathbf{B}}-I+\gamma^{-2} \mathbf{D}_{i 2}^{T} \mathbf{D}_{i 2} & \overline{\mathbf{B}}^{T} \mathbf{P}_{j} \overline{\mathbf{A}}+\gamma^{-2} \mathbf{D}_{i 2}^{T} \overline{\mathbf{C}} & \overline{\mathbf{B}}^{T} \mathbf{P}_{j} \overline{\mathbf{b}}_{i} \\
* & \overline{\mathbf{A}}^{T} \mathbf{P}_{j} \overline{\mathbf{A}}-\overline{\mathbf{E}}^{T} \mathbf{P}_{i} \overline{\mathbf{E}}+\gamma^{-2} \overline{\mathbf{C}}^{T} \overline{\mathbf{C}} & \overline{\mathbf{A}}^{T} \mathbf{P}_{j} \overline{\mathbf{b}}_{i} \\
* & * & \overline{\mathbf{b}}_{i}^{T} \mathbf{P}_{j} \overline{\mathbf{b}}_{i}
\end{array}\right] \\
& \times\left[\begin{array}{c}
\mathbf{w}(k) \\
\widetilde{\mathbf{x}}(k) \\
1
\end{array}\right]<0 .
\end{aligned}
$$

From (46), we get

$$
\overline{\mathbf{A}}^{T} \mathbf{P}_{j} \overline{\mathbf{A}}-\overline{\mathbf{E}}^{T} \mathbf{P}_{i} \overline{\mathbf{E}}+\gamma^{-2} \overline{\mathbf{C}}^{T} \overline{\mathbf{C}}<0 .
$$

From the proof of Theorem 7 , it is easy to see that $(\overline{\mathbf{E}}, \overline{\mathbf{A}})$ is causal. Thus the regularity of $(\overline{\mathbf{E}}, \overline{\mathbf{A}})$ is implied. As a result, the closed-loop system (15) is said to be admissible.

Remark 11. According to the proof of Theorem 7, we use the same method to get the regularity of $(\overline{\mathbf{E}}, \overline{\mathbf{A}})$. We also multiply (27) by the grade 1 eigenvectors $\boldsymbol{\nu}^{1}$ and its Hermitian $\boldsymbol{\nu}^{1^{*}}$, respectively. In view of Definition 3, replacing $\overline{\mathrm{A}} \boldsymbol{\nu}^{1}$ by $\mathbf{E} \nu^{2}$ and noting that $\mathbf{E} \boldsymbol{v}^{1}=0$, as a result, the closed-loop system (15) is said to be admissible.

Then, by taking into consideration the partition information (9) and applying the S-procedure, we have that the following inequality implies (46) with $\lambda_{i j}<0, i \in I_{1},(i, j) \in$ $\Omega$ :

$$
\begin{aligned}
& {\left[\begin{array}{c}
\mathbf{w}(k) \\
\widetilde{\mathbf{x}}(k) \\
1
\end{array}\right]^{T}} \\
& \times\left\{\left[\begin{array}{l}
\overline{\mathbf{B}}^{T} \\
\overline{\mathbf{A}}^{T} \\
\overline{\mathbf{b}}_{i}^{T}
\end{array}\right] \mathbf{P}_{j}\left({ }^{*}\right)+\gamma^{-2}\left[\begin{array}{c}
\mathbf{D}_{i 2}^{T} \\
\overline{\mathbf{C}}^{T} \\
\mathbf{0}
\end{array}\right]\left({ }^{*}\right)\right. \\
& \left.+\left[\begin{array}{ccc}
-\mathbf{I} & \mathbf{0} & \mathbf{0} \\
* & -\overline{\mathbf{E}}^{T} \mathbf{P}_{i} \overline{\mathbf{E}}+\lambda_{i j} \overline{\mathbf{F}}_{i}^{T} \overline{\mathbf{F}}_{i} & \lambda_{i j} \overline{\mathbf{F}}_{i}^{T} f_{i} \\
* & * & \lambda_{i j}\left(f_{i}^{T} f_{i}-1\right)
\end{array}\right]\right\} \\
& \times\left[\begin{array}{c}
\mathbf{w}(k) \\
\widetilde{\mathbf{x}}(k) \\
1
\end{array}\right]<0, \quad(i, j) \in \Omega .
\end{aligned}
$$

By using the well-known Schur complement, it is easy to see that the following inequality implies (48):

$$
\left.\begin{array}{ccccc}
-\gamma^{2} \mathbf{I} & \mathbf{0} & \mathbf{D}_{i 2} & \overline{\mathbf{C}} & \mathbf{0} \\
* & -\mathbf{P}_{j}^{-1} & \overline{\mathbf{B}} & \overline{\mathbf{A}} & \overline{\mathbf{b}_{i}} \\
* & * & -\mathbf{I} & \mathbf{0} & \mathbf{0} \\
* & * & * & -\overline{\mathbf{E}}^{T} \mathbf{P}_{i} \overline{\mathbf{E}}+\lambda_{i j} \overline{\mathbf{F}}_{i}^{T} \overline{\mathbf{F}}_{i} & \lambda_{i j} \overline{\mathbf{F}}_{i}^{T} f_{i} \\
* & * & * & * & \lambda_{i j}\left(f_{i}^{T} f_{i}-1\right)
\end{array}\right]
$$

On the other hand, by using the relations given in (3), (49) can be easily rewritten as follows: 
It is easy to see that the following inequality implies (50):

$$
\begin{aligned}
& {\left[\begin{array}{ccccccc}
-\gamma^{2} \mathbf{I} & \mathbf{0} & \mathbf{0} & \mathbf{D}_{i 2} & \mathbf{F}_{i}-\mathbf{D}_{f} \mathbf{C}_{i} & \mathbf{G}_{i} \widehat{\mathbf{Q}}_{i s}+\mathbf{G}_{i} \overline{\mathbf{W}_{i s}} \mathbf{H}_{i}+\mathbf{G}_{i} \mathbf{W}_{i s} \Delta \mathbf{K}_{i}-\mathbf{C}_{f} & \mathbf{0} \\
* & \mathbf{H}_{1} & \mathbf{H}_{2} & \mathbf{D}_{i 1} & \mathbf{A}_{i} & \mathbf{B}_{i} \widehat{\mathbf{Q}}_{i s}+\mathbf{B}_{i} \overline{\mathbf{W}_{i s}} \mathbf{H}_{i}+\mathbf{B}_{i} \mathbf{W}_{i s} \Delta \mathbf{K}_{i} & \mathbf{E b}_{i} \\
* & * & \mathbf{H}_{3} & \mathbf{0} & \mathbf{B}_{f} \mathbf{C}_{i} & \mathbf{A}_{f} & \mathbf{0} \\
* & * & * & -\mathbf{I} & \mathbf{0} & \mathbf{0} & \mathbf{0} \\
* & * & * & * & J_{1} & J_{2} & I_{1} \\
* & * & * & * & * & J_{3} & I_{2} \\
* & * & * & * & * & * & \lambda_{i j}\left(f_{i}^{T} f_{i}-1\right)
\end{array}\right]} \\
& +\operatorname{sym}\left\{\left[\begin{array}{c}
\mathbf{0} \\
\mathbf{W}_{i 1} \\
\mathbf{0} \\
\mathbf{0} \\
\mathbf{0} \\
\mathbf{0} \\
\mathbf{0}
\end{array}\right]\left[\begin{array}{lllllll}
\mathbf{0} & \mathbf{0} & \mathbf{0} & \mathbf{0} & \mathbf{E}_{i 1} & \mathbf{0} & \mathbf{E}_{i 2}
\end{array}\right]\right\}<0, \quad(i, j) \in \Omega \text {. }
\end{aligned}
$$

From the proof of Theorem 7, based on Lemma 6, by introducing three sets of positive scalar parameters $\varepsilon_{i j}>0$, $\varepsilon_{i j}^{\prime}>0$, and $\varepsilon_{i j}^{\prime \prime}>0, i \in I_{1},(i, j) \in \Omega$, it is easy to see that the following inequality implies (51):

$$
\left.\begin{array}{cccccccccc}
-\varepsilon_{i j}^{\prime \prime} \mathbf{I}_{s 2} & \mathbf{0} & \mathbf{0} & \mathbf{0} & \mathbf{0} & \mathbf{0} & \mathbf{0} & \mathbf{0} & \mathbf{E}_{i 3} & \mathbf{0} \\
* & -\varepsilon_{i j}^{\prime} \mathbf{I}_{s 2} & \mathbf{0} & \mathbf{0} & \mathbf{0} & \mathbf{0} & \mathbf{0} & \mathbf{0} & \mathbf{E}_{i 3} & \mathbf{0} \\
* & * & -\varepsilon_{i j} \mathbf{I}_{s 2}+\delta & \mathbf{0} & \chi & \mathbf{0} & \mathbf{0} & \mathbf{E}_{i 1}+\lambda_{i j}^{-1} \mathbf{E}_{i 2} I_{1}^{T} & \lambda_{i j}^{-1} \mathbf{E}_{i 2} I_{2}^{T} & \mathbf{E}_{i 2} \\
* & * & * & \phi & \mathbf{0} & \mathbf{0} & \mathbf{D}_{i 2} & \mathbf{F}_{i}-\mathbf{D}_{f} \mathbf{C}_{i} & \mathcal{2} & \mathbf{0} \\
* & * & * & * & \wp & \mathbf{H}_{2} & \mathbf{D}_{i 1} & \mathbf{A}_{i}+\lambda_{i j}^{-1} \mathbf{E b}_{i} I_{1}^{T} & \mathfrak{\Im} & \mathbf{E b}_{i} \\
* & * & * & * & * & \mathbf{H}_{3} & \mathbf{0} & \mathbf{B}_{f} \mathbf{C}_{i} & \mathbf{A}_{f} & \mathbf{0} \\
* & * & * & * & * & * & -\mathbf{I} & \mathbf{0} & \mathbf{0} & \mathbf{0} \\
* & * & * & * & * & * & * & J_{1}+\lambda_{i j}^{-1} I_{1} I_{1}^{T} & J_{2}+\lambda_{i j}^{-1} I_{1} I_{2}^{T} & I_{1} \\
* & * & * & * & * & * & * & * & J_{3}+\lambda_{i j}^{-1} I_{2} I_{2}^{T} & I_{2} \\
* & * & * & * & * & * & * & * & * & \lambda_{i j}\left(f_{i}^{T} f_{i}-I\right)
\end{array}\right]
$$

where $\boldsymbol{\alpha}=\mathbf{G}_{i} \mathbf{W}_{i s} \mathbf{W}_{i 1}, \boldsymbol{\varphi}=\mathbf{B}_{i} \mathbf{W}_{i s} \mathbf{W}_{i 1}, \boldsymbol{\wp}=\mathbf{H}_{1}+\varepsilon_{i j} \mathbf{W}_{i 1} \mathbf{W}_{i 1}^{T}+$ $\varepsilon_{i j}^{\prime \prime} \boldsymbol{\varphi} \boldsymbol{\varphi}^{T}+\lambda_{i j}^{-1} \mathbf{E b}_{i}\left(\mathbf{E b}_{i}\right)^{T}, \mathfrak{\Im}=\mathbf{B}_{i} \widehat{\mathbf{Q}}_{i s}+\mathbf{B}_{i} \overline{\mathbf{W}_{i s}} \mathbf{H}_{i}+\lambda_{i j}^{-1} \mathbf{E} \mathbf{b}_{i} I_{2}^{T}, \boldsymbol{N}=$ $\mathbf{G}_{i} \widehat{\mathbf{Q}}_{i s}+\mathbf{G}_{i} \overline{\mathbf{W}_{i s}} \mathbf{H}_{i}-\mathbf{C}_{f}, \chi=\lambda_{i j}^{-1} \mathbf{E}_{i 2}\left(\mathbf{E b}_{i}\right)^{T}, \phi=-\gamma^{2} \mathbf{I}+\varepsilon_{i j}^{\prime} \boldsymbol{\alpha} \boldsymbol{\alpha}^{T}$, and $\delta=\lambda_{i j}^{-1} \mathbf{E}_{i 2} \mathbf{E}_{i 2}^{T}$.
On the other hand, it follows that $\mathbf{b}_{i}+\Delta \mathbf{b}_{i}=0$; it is easy to see the following inequality:

$$
\left[\begin{array}{ccccccccc}
-\varepsilon_{i j}^{\prime \prime} \mathbf{I}_{s 2} & \mathbf{0} & \mathbf{0} & \mathbf{0} & \mathbf{0} & \mathbf{0} & \mathbf{0} & \mathbf{0} & \mathbf{E}_{i 3} \\
* & -\varepsilon_{i j}^{\prime} \mathbf{I}_{s 2} & \mathbf{0} & \mathbf{0} & \mathbf{0} & \mathbf{0} & \mathbf{0} & \mathbf{0} & \mathbf{E}_{i 3} \\
* & * & -\varepsilon_{i j} \mathbf{I}_{s 2} & \mathbf{0} & \mathbf{0} & \mathbf{0} & \mathbf{0} & \mathbf{E}_{i 1} & \mathbf{0} \\
* & * & * & \phi & \mathbf{0} & \mathbf{0} & \mathbf{D}_{i 2} & \mathbf{F}_{i}-\mathbf{D}_{f} \mathbf{C}_{i} & \mathbf{G}_{i} \widehat{\mathbf{Q}}_{i s}+\mathbf{G}_{i} \overline{\mathbf{W}_{i s}} \mathbf{H}_{i}-\mathbf{C}_{f} \\
* & * & * & * & \sigma & \mathbf{H}_{2} & \mathbf{D}_{i 1} & \mathbf{A}_{i} & \mathbf{B}_{i} \widehat{\mathbf{Q}}_{i s}+\mathbf{B}_{i} \mathbf{W}_{i s} \mathbf{H}_{i} \\
* & * & * & * & * & \mathbf{H}_{3} & \mathbf{0} & \mathbf{B}_{f} \mathbf{C}_{i} & \mathbf{A}_{f} \\
* & * & * & * & * & * & -\mathbf{I} & \mathbf{0} & \mathbf{0} \\
* & * & * & * & * & * & * & J_{1} & J_{2} \\
* & * & * & * & * & * & * & * & J_{3}
\end{array}\right]<0, \quad(i, j) \in \Omega,
$$

where $\sigma=\mathbf{H}_{1}+\varepsilon_{i j} \mathbf{W}_{i 1} \mathbf{W}_{i 1}^{T}+\varepsilon_{i j}^{\prime \prime} \boldsymbol{\varphi} \boldsymbol{\varphi}^{T}$. 
On the other hand, from the proof of Theorem 7, it is clear that all trajectories of system (1) starting from the origin will remain inside the region $\cup_{i \in I}\left(\varepsilon\left(\mathbf{P}_{i}, \beta\right) \cap \Re_{i}\right)$. This completes the proof.

For a fixed scalar $\beta \leq \beta_{M}$, the smallest $\mathrm{H}$-infinity performance $\gamma$ can be measured by solving this optimization problem: ( $\left.\min / \mathbf{P}_{i}^{-1}, \mathbf{Y}_{i}, \mathbf{Z}_{i}\right) \gamma$, so that the LMIs in 10 hold.

\section{Numerical Examples}

Example 1. To illustrate the analysis and synthesis methods described in the previous sections, we consider the stabilization problem for system (1) with the following data:

$$
\begin{aligned}
& \mathbf{A}_{1}=\left[\begin{array}{lll}
0.1432 & 0.2312 & 0.4232 \\
0.2123 & 0.6765 & 0.3454 \\
1.0001 & 2.0001 & 0.2281
\end{array}\right], \\
& \mathbf{A}_{2}=\left[\begin{array}{lll}
0.2343 & 0.3343 & 0.1565 \\
1.0000 & 0.5232 & 0.6456 \\
1.2131 & 0.5456 & 0.8564
\end{array}\right] \\
& \mathbf{B}_{1}=\left[\begin{array}{c}
-0.3 \\
0.2 \\
1.0
\end{array}\right], \quad \mathbf{B}_{2}=\left[\begin{array}{l}
0.1 \\
0.2 \\
0.5
\end{array}\right], \\
& \mathbf{G}_{1}=\left[\begin{array}{c}
-0.5 \\
0.4 \\
1.0
\end{array}\right], \quad \mathbf{G}_{2}=\left[\begin{array}{c}
-0.1 \\
0.2 \\
1.0
\end{array}\right] \\
& \mathbf{D}_{11}=\left[\begin{array}{lll}
1.1 & 2.3 & 1.0 \\
1.3 & 1.0 & 2.9 \\
2.0 & 0.2 & 0.4
\end{array}\right], \quad \mathbf{D}_{12}=\left[\begin{array}{lll}
0.1 & 1.3 & 3.0 \\
1.2 & 2.2 & 0.3 \\
0.5 & 4.1 & 4.3
\end{array}\right] \\
& \mathbf{D}_{21}=\left[\begin{array}{lll}
1.0 & 3.8 & 1.1 \\
3.0 & 1.9 & 2.3 \\
1.0 & 0.2 & 0.4
\end{array}\right], \quad \mathbf{D}_{22}=\left[\begin{array}{lll}
1.7 & 0.1 & 1.2 \\
1.3 & 3.0 & 2.0 \\
2.0 & 0.2 & 0.4
\end{array}\right], \\
& \mathrm{C}_{1}=\left[\begin{array}{lll}
1 & 2 & 1 \\
2 & 1 & 2 \\
1 & 4 & 3
\end{array}\right], \quad \mathbf{C}_{2}=\left[\begin{array}{lll}
2 & 1 & 3 \\
3 & 1 & 2 \\
4 & 2 & 1
\end{array}\right] \\
& \mathbf{E}=\left[\begin{array}{ccc}
1 & 1 & 1 \\
0 & 0 & 0 \\
0 & 0 & 0
\end{array}\right], \quad \mathbf{b}_{1}=\left[\begin{array}{c}
0.05 \\
0.05 \\
1.00
\end{array}\right] \\
& \mathbf{b}_{2}=\left[\begin{array}{c}
1.00 \\
-1.00 \\
0.50
\end{array}\right], \quad \mathbf{W}_{11}=\left[\begin{array}{l}
0.00 \\
0.03 \\
1.00
\end{array}\right] \\
& \mathbf{W}_{21}=\left[\begin{array}{l}
0.00 \\
0.02 \\
2.00
\end{array}\right], \quad \mathbf{E}_{11}=\left[\begin{array}{l}
0.00 \\
0.02 \\
1.00
\end{array}\right]^{T} \text {, } \\
& \mathbf{E}_{12}=0.03, \quad \mathbf{E}_{21}=\left[\begin{array}{c}
0 \\
0.01 \\
3
\end{array}\right]^{T} \text {, }
\end{aligned}
$$

$$
\begin{gathered}
\mathbf{E}_{22}=0.04, \quad \mathbf{E}_{13}=\left[\begin{array}{l}
0.40 \\
0.02 \\
1.00
\end{array}\right]^{T}, \\
\mathbf{E}_{23}=\left[\begin{array}{l}
0.50 \\
0.02 \\
1.00
\end{array}\right]^{T} .
\end{gathered}
$$

Saturation level $\overline{\mathbf{u}}=6$, and system state is bounded by (5) with $\mathbf{L}=I$ and $\overline{\mathbf{g}}=\left[\begin{array}{lll}30 & 30 & 30\end{array}\right]^{T}$. It is assumed that

$$
\begin{aligned}
& \mathfrak{R}_{1}=\left\{\mathbf{x}(t) \mid 5 \leq\left[\begin{array}{lll}
1 & 0 & 0
\end{array}\right] \mathbf{x}(t) \leq 30\right\} \\
& \mathfrak{R}_{2}=\left\{\mathbf{x}(t) \mid-30 \leq\left[\begin{array}{lll}
1 & 0 & 0
\end{array}\right] \mathbf{x}(t) \leq-5\right\}
\end{aligned}
$$

Let the coefficient matrices of ellipsoid be given as

$$
\begin{array}{ll}
\mathbf{F}_{1}=2 \times \frac{\mathbf{C}_{1}^{T}}{\left(\beta_{1}-\alpha_{1}\right)}, & f_{1}=-\frac{\beta_{1}+\alpha_{1}}{\beta_{1}-\alpha_{1}}, \\
\mathbf{F}_{2}=2 \times \frac{\mathbf{C}_{2}^{T}}{\left(\beta_{2}-\alpha_{2}\right)}, & f_{2}=-\frac{\beta_{2}+\alpha_{2}}{\beta_{2}-\alpha_{2}},
\end{array}
$$

where $\alpha_{1}=3, \beta_{1}=10, \alpha_{2}=4$, and $\beta_{2}=9$.

Let the interference signal be given as $\mathbf{w}(k)=e^{-5 k}$.

With these parameters solving LMIs, we found that they are feasible and the solution is as follows.

(A) Resilient Output Feedback Control of the Discrete-Time Singular Piecewise-Affine System. By applying Theorem 7 directly, one can obtain

$$
\begin{aligned}
& \mathbf{K}_{1}=\left[\begin{array}{lll}
-30.8973 & -17.0674 & -20.5996
\end{array}\right], \\
& \mathbf{K}_{2}=\left[\begin{array}{lll}
18.9530 & 15.1858 & 10.1835
\end{array}\right] \\
& \mathbf{P}_{1}=\left[\begin{array}{ccc}
3.0269 & -1.5503 & 2.5663 \\
-1.5503 & 4.8114 & -7.1203 \\
2.5663 & -7.1203 & 0.8454
\end{array}\right], \\
& \mathbf{P}_{2}=\left[\begin{array}{ccc}
5.0239 & -4.5333 & 10.5123 \\
-4.5333 & 5.4514 & -1.1113 \\
10.5123 & -1.1113 & 7.8674
\end{array}\right]
\end{aligned}
$$

The $\mathrm{H}$-infinity performance $\gamma=6.9025$.

From the above two sets of Lyapunov matrices, Figure 2 presents the estimated domains of attraction of system (12), which is bounded by the solid line.

(B) Resilient Filter for Discrete-Time Singular Piecewise-Affine Systems with Uncertain Parameters. Consider

$$
\begin{aligned}
& K_{1}=\left[\begin{array}{lll}
5.2373 & 6.0612 & 1.5916
\end{array}\right], \\
& K_{2}=\left[\begin{array}{lll}
-3.9530 & -6.1848 & -0.8335
\end{array}\right],
\end{aligned}
$$




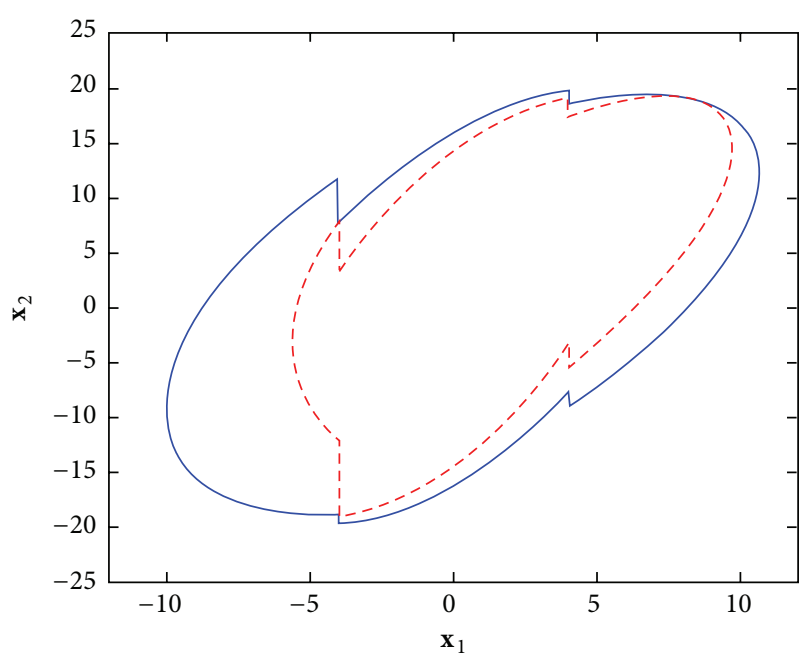

FIGURE 2: Estimated domains of attraction in Example 1.

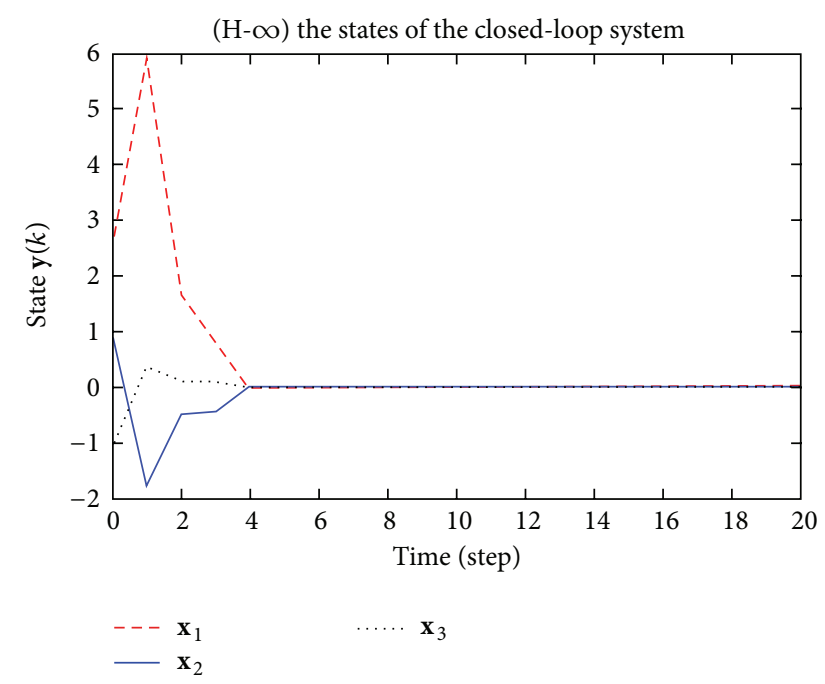

FIGURE 3: State responses of the closed-loop system.

and the $\mathrm{H}$-infinity performance $\gamma=145.6232$. Consider

$$
\begin{aligned}
& A_{f}=\left[\begin{array}{ccc}
7.7703 & -8.0816 & 0.0444 \\
-6.6017 & 1.688 & 0.5420 \\
7.0078 & -1.0010 & 1.6448
\end{array}\right], \\
& B_{f}=\left[\begin{array}{ccc}
3.2033 & 7.0812 & -0.4566 \\
0.0821 & 5.0721 & -7.1028 \\
5.3240 & -4.1035 & 5.2551
\end{array}\right], \\
& C_{f}=\left[\begin{array}{ccc}
7.1427 & 2.9229 & 0.8533 \\
-1.3315 & 14.3578 & -4.6118 \\
-8.4661 & 4.3829 & 1.2413
\end{array}\right], \\
& D_{f}=\left[\begin{array}{ccc}
-0.0336 & -0.0403 & 0.0559 \\
8.0976 & 4.0324 & -0.8279 \\
-0.1886 & 6.2133 & 8.2879
\end{array}\right] .
\end{aligned}
$$

Example 2. Now, we consider a practical physical example to illustrate the analysis and synthesis methods described in the previous sections; we consider the stabilization problem for system (1) with the tunnel diode circuit [47]. Some more details on the dynamics of the tunnel diode circuit in Example 2 are given in [47]. For this system, it is assumed that the controller is subject to saturation constraint with $\overline{\mathbf{u}}=6$ and the system state is bounded by (5) with $\mathbf{L}=I$ and $\overline{\mathbf{g}}=\left[\begin{array}{lll}30 & 30 & 30\end{array}\right]^{T} \cdot \mathbf{x}_{1}(k)$ can be exactly described by capacitor voltage, $\mathbf{x}_{2}(k)$ can be exactly described by inductive current, and $\mathbf{x}_{3}(k)$ can be exactly described by the current which flows through tunnel diode. The tunnel diode circuit can be abstracted as a system which is described by the following dynamics:

$$
\begin{gathered}
\mathbf{E x}(k+1)=\left(\mathbf{A}_{i}+\Delta \mathbf{A}_{i}\right) \mathbf{x}(k)+\mathbf{B}_{i} \operatorname{sat}(\mathbf{u}(k)) \\
+\mathbf{D}_{i 1} \mathbf{w}(k)+\mathbf{E}\left(\mathbf{b}_{i}+\Delta \mathbf{b}_{i}\right), \\
\mathbf{y}(k)=\mathbf{C}_{i} \mathbf{x}(k), \quad i=1,2 .
\end{gathered}
$$

The data we need is given previously, where $\beta_{M}=5.6567$. Now, we also need some data: $\beta=5.0 \leq \beta_{M}$.

The gain matrices for resilient $\mathrm{H}$-infinity output feedback controller are obtained:

$$
\begin{aligned}
& \mathbf{K}_{1}=\left[\begin{array}{lll}
-14.2233 & 0.3412 & 11.5316
\end{array}\right] \\
& \mathbf{K}_{2}=\left[\begin{array}{lll}
30.9430 & -6.2848 & -45.2235
\end{array}\right] .
\end{aligned}
$$

The $\mathrm{H}$-infinity performance $\gamma=7.5744$.

The design parameters are obtained:

$$
\begin{aligned}
& \mathbf{A}_{f}=\left[\begin{array}{ccc}
14.5503 & 2.4416 & 3.5644 \\
-4.6417 & 21.4488 & 4.5420 \\
9.0898 & -7.7010 & 6.6468
\end{array}\right], \\
& \mathbf{B}_{f}=\left[\begin{array}{ccc}
2.3233 & 5.0662 & -7.4666 \\
0.0121 & 1.0121 & -23.1228 \\
1.3240 & 34.1335 & 25.2651
\end{array}\right], \\
& \mathbf{C}_{f}=\left[\begin{array}{ccc}
1.1421 & -1.9229 & 3.8233 \\
-6.3615 & 13.3528 & -12.6138 \\
-4.4561 & 1.3869 & 21.2113
\end{array}\right], \\
& \mathbf{D}_{f}=\left[\begin{array}{ccc}
-7.0666 & 2.0223 & 1.0259 \\
-8.0976 & -4.3324 & -4.4279 \\
2.1836 & 5.2533 & 5.2859
\end{array}\right] .
\end{aligned}
$$

\section{Conclusions}

In this paper, we have proposed new LMI conditions for the problem of designing robust $\mathrm{H}$-infinity output feedback controller and resilient filtering for a class of discretetime singular piecewise-affine systems with input saturation and state constraints, involving norm-bounded time-varying parameters uncertainties. Based on a singular piecewise Lyapunov function combined with S-procedure and some matrix inequality convexifying techniques, the controller gains and the filter design parameters have been obtained by solving a family of LMIs. Meanwhile, by presenting the corresponding optimization methods, the domain of attraction and the disturbance tolerance level is maximized, 
and the $\mathrm{H}$-infinity performance $\gamma$ is minimized. Simulation examples are presented to demonstrate the effectiveness and practicability of the proposed approaches.

As for further studies, the piecewise-affine controller will be used to investigate a similar robust stabilization problem of continuous-time singular piecewise-affine systems. In addition, resilient $\mathrm{H}$-infinity guaranteed cost controller will be investigated for singular piecewise-affine systems with input saturation and state constraints.

\section{Conflict of Interests}

The authors declare that there is no conflict of interests regarding the publication of this paper.

\section{Acknowledgment}

This research is sponsored by the National Natural Science Foundation of China (Grant no. 61004038).

\section{References}

[1] S. Mirzazad-Barijough and J.-W. Lee, "Stability and transient performance of discrete-time piecewise affine systems," IEEE Transactions on Automatic Control, vol. 57, no. 4, pp. 936-949, 2012.

[2] S. Mirzazad-Barijough and J. W. Lee, "Robust stability and performance analysis of discrete-time piecewise affine systems with disturbances," in Proceedings of the IEEE 51st Annual Conference on Decision and Control (CDC '12), pp. 4229-4234, 2012.

[3] M. Rubagotti, L. Zaccarian, and A. Bemporad, "Stability analysis of discrete-time piecewise-affine systems over non-invariant domains," in Proceedings of the 51st IEEE Conference on Decision and Control (CDC '12), pp. 4235-4240, Maui, Hawaii, USA, December 2012.

[4] N. Eghbal, N. Pariz, and A. Karimpour, "Discontinuous piecewise quadratic Lyapunov functions for planar piecewise affine systems," Journal of Mathematical Analysis and Applications, vol. 399, no. 2, pp. 586-593, 2013.

[5] M. Rubagotti, S. Trimboli, and A. Bemporad, "Stability and invariance analysis of uncertain discrete-time piecewise affine systems," IEEE Transactions on Automatic Control, vol. 58, no. 9, pp. 2359-2365, 2013.

[6] A. Bemporad, G. Ferrari-Trecate, and M. Morari, "Observability and controllability of piecewise affine and hybrid systems," IEEE Transactions on Automatic Control, vol. 45, no. 10, pp. 18641876, 2000.

[7] W. P. Heemels, M. K. Camlibel, and J. M. Schumacher, "On the dynamic analysis of piecewise-linear networks," IEEE Transactions on Circuits and Systems. I. Fundamental Theory and Applications, vol. 49, no. 3, pp. 315-327, 2002.

[8] J. Imura, "Classification and stabilizability analysis of bimodal piecewise affine systems," International Journal of Robust and Nonlinear Control, vol. 12, no. 10, pp. 897-926, 2002.

[9] M. Johansson and A. Rantzer, "Computation of piecewise quadratic Lyapunov functions for hybrid systems," IEEE Transactions on Automatic Control, vol. 43, no. 4, pp. 555-559, 1998.

[10] A. Rantzer and M. Johansson, "Piecewise linear quadratic optimal control," IEEE Transactions on Automatic Control, vol. 45 , no. 4, pp. 629-637, 2000.
[11] M. Johansson, Piecewise Linear Control Systems-A Computational Approach, vol. 284, 2003.

[12] J. Imura and A. van der Schaft, "Characterization of wellposedness of piecewise-linear systems," IEEE Transactions on Automatic Control, vol. 45, no. 9, pp. 1600-1619, 2000.

[13] G. Feng, "Controller design and analysis of uncertain piecewiselinear systems," IEEE Transactions on Circuits and Systems. I. Fundamental Theory and Applications, vol. 49, no. 2, pp. 224232, 2002.

[14] Y. Zhu, D. Q. Li, and G. Feng, "H-infinity controller synthesis of uncertain piecewise continuous-time linear systems," IEE Proceedings-Control Theory and Applications, vol. 152, pp. 513519, 2005.

[15] J. X. Zhang and W. S. Tang, "Output feedback H(infinity) control for uncertain piecewise linear systems," Journal of Dynamical and Control Systems, vol. 14, no. 1, pp. 121-144, 2008.

[16] J. Zhang and W. Tang, "Output feedback optimal guaranteed cost control of uncertain piecewise linear systems," International Journal of Robust and Nonlinear Control, vol. 19, no. 5, pp. 569-590, 2009.

[17] G. Bianchini, S. Paoletti, and A. Vicino, "Convex relaxations for $L_{2}$-gain analysis of piecewise affine/polynomial systems," International Journal of Control, vol. 86, no. 7, pp. 1207-1213, 2013.

[18] M. di Bernardo, U. Montanaro, and S. Santini, "Hybrid model reference adaptive control of piecewise affine systems," IEEE Transactions on Automatic Control, vol. 58, no. 2, pp. 304-316, 2013.

[19] W. P. M. H. Heemels, M. Lazar, N. Van De Wouw, and A. Pavlov, "Observer-based control of discrete-time piecewise affine systems: exploiting continuity twice," in Proceedings of the 47th IEEE Conference on Decision and Control (CDC '08), pp. 4675-4680, Cancún, Mexico, December 2008.

[20] G. Pola and M. D. Di Benedetto, "Symbolic models and control of discrete-time piecewise affine systems: an approximate simulation approach," IEEE Transactions on Automatic Control, vol. 59, no. 1, pp. 175-180, 2014.

[21] L. Rodrigues and J. P. How, "Observer-based control of piecewise-affine systems," International Journal of Control, vol. 76, no. 5, pp. 459-477, 2003.

[22] A. Hassibi and S. Boyd, "Quadratic stabilization and control of piecewise-linear systems," in Proceedings of the American Control Conference (ACC '98), vol. 6, pp. 3659-3664, Philadelphia, $\mathrm{Pa}$, USA, June 1998.

[23] A. L. Juloski, W. P. Heemels, and S. Weiland, "Observer design for a class of piecewise linear systems," International Journal of Robust and Nonlinear Control, vol. 17, no. 15, pp. 1387-1404, 2007.

[24] S. Pettersson and B. Lennartson, "Hybrid system stability and robustness verification using linear matrix inequalities," International Journal of Control, vol. 75, no. 16-17, pp. 1335-1355, 2002.

[25] L. Gao and Y. Wu, "A LMI-based approach for robust stabilization of uncertain linear continuous time singular Markov switched systems with time delays," in Proceedings of the 25th Chinese Control Conference (CCC '06), pp. 844-849, IEEE, Harbin, China, August 2006.

[26] G. Zong, Y. Tian, and H. Sun, "Stabilization for a class of piecewise continuous-time switched control systems," in Proceedings of the IEEE 7th World Congress on Intelligent Control and Automation (WCICA '08), vol. 1-23, pp. 7226-7229, June 2008. 
[27] G. Feng, "Observer-based output feedback controller design of piecewise discrete-time linear systems," IEEE Transactions on Circuits and Systems. I: Fundamental Theory and Applications, vol. 50, no. 3, pp. 448-451, 2003.

[28] H. Lin and P. J. Antsaklis, "Hybrid state feedback stabilization with $l_{2}$ performance for disrete-time switched linear systems," International Journal of Control, vol. 81, no. 7, pp. 1114-1124, 2008.

[29] J. Xu, L. H. Xie, and G. Feng, "Feedback control design for discrete-time piecewise affine systems," in Proceedings of the International Conference on Control and Automation (ICCA '05), vol. 1, pp. 425-430, IEEE, June 2005.

[30] T. Zhang and G. Feng, "Output tracking of piecewise-linear systems via error feedback regulator with application to synchronization of nonlinear Chua's circuit," IEEE Transactions on Circuits and Systems I: Regular Papers, vol. 54, no. 8, pp. 18521863, 2007.

[31] Y. L. Wei, M. Wang, and J. B. Qiu, "New approach to delaydependent $H_{\infty}$ filtering for discrete-time Markovian jump systems with time-varying delay and incomplete transition descriptions," IET Control Theory \& Applications, vol. 7, no. 5, pp. 684-696, 2013.

[32] P. Shi, E.-K. Boukas, and R. K. Agarwal, "Kalman filtering for continuous-time uncertain systems with Markovian jumping parameters," IEEE Transactions on Automatic Control, vol. 44, no. 8, pp. 1592-1597, 1999.

[33] C.-H. Lien, J.-D. Chen, C.-T. Lee, R.-S. Chen, and C.-D. Yang, "Robust $H_{\infty}$ filter design for discrete-time switched systems with interval time-varying delay and linear fractional perturbations: LMI optimization approach," Applied Mathematics and Computation, vol. 219, no. 24, pp. 11395-11407, 2013.

[34] L. Y. Chung, C. H. Lien, K. W. Yu, and J. D. Chen, "Robust $H_{\infty}$ filtering for discrete switched systems with interval timevarying delay," Signal Processing, vol. 94, no. 1, pp. 661-669, 2014.

[35] G.-H. Yang and J. L. Wang, "Robust nonfragile Kalman filtering for uncertain linear systems with estimator gain uncertainty," IEEE Transactions on Automatic Control, vol. 46, no. 2, pp. 343348, 2001.

[36] G. Song and Z. Wang, "A delay partitioning approach to output feedback control for uncertain discrete time-delay systems with actuator saturation," Nonlinear Dynamics, vol. 74, no. 1-2, pp. 189-202, 2013.

[37] S. Huang, Z. Xiang, and H. R. Karimi, "Robust $l_{2}$-gain control for $2 \mathrm{D}$ nonlinear stochastic systems with time-varying delays and actuator saturation," Journal of the Franklin Institute, vol. 350, no. 7, pp. 1865-1885, 2013.

[38] J. Dong and G. H. Yang, "Static output feedback control synthesis for linear systems with time-invariant parametric uncertainties," IEEE Transactions on Automatic Control, vol. 52, no. 10, pp. 1930-1936, 2007.

[39] B. Zhou, Z. Lin, and G.-R. Duan, "A parametric Lyapunov equation approach to low gain feedback design for discrete-time systems," Automatica, vol. 45, no. 1, pp. 238-244, 2009.

[40] T. Hu, Z. Lin, and B. M. Chen, "Analysis and design for discretetime linear systems subject to actuator saturation," Systems and Control Letters, vol. 45, no. 2, pp. 97-112, 2002.

[41] T. Hu, Z. Lin, and B. M. Chen, "An analysis and design method for linear systems subject to actuator saturation and disturbance," Automatica, vol. 38, no. 2, pp. 351-359, 2002.
[42] J. Qiu, T. Zhang, G. Feng, and H. Liu, "Piecewise affine modelbased $H_{\infty}$ static output feedback control of constrained nonlinear processes," IET Control Theory \& Applications, vol. 4, no. 11, pp. 2315-2330, 2010.

[43] Y. Gao, Z. Liu, and H. Chen, "Robust $H_{\infty}$ control for constrained discrete-time piecewise affine systems with timevarying parametric uncertainties," IET Control Theory \& Applications, vol. 3, no. 8, pp. 1132-1144, 2009.

[44] Y. Chen, Q. Zhou, and S. Fei, "Robust stabilization and $l_{2}$-gain control of uncertain discrete-time constrained piecewise-affine systems," Nonlinear Dynamics, vol. 75, no. 1-2, pp. 127-140, 2014.

[45] K. L. Hsiung and L. Lee, "Lyapunov inequality and bounded real lemma for discrete-time descriptor systems," IEE ProceedingsControl Theory and Applications, vol. 146, no. 4, pp. 327-331, 1999.

[46] J. Qiu, G. Feng, and H. Gao, "Approaches to robust $H_{\infty}$ static output feedback control of discrete-time piecewise-affine systems with norm-bounded uncertainties," International Journal of Robust and Nonlinear Control, vol. 21, no. 7, pp. 790-814, 2011.

[47] L. Rodrigues and S. Boyd, "Piecewise-affine state feedback for piecewise-affine slab systems using convex optimization," Systems \& Control Letters, vol. 54, no. 9, pp. 835-853, 2005. 


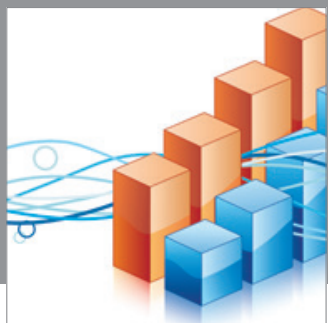

Advances in

Operations Research

mansans

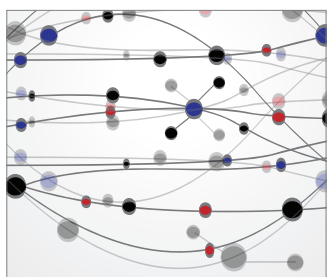

The Scientific World Journal
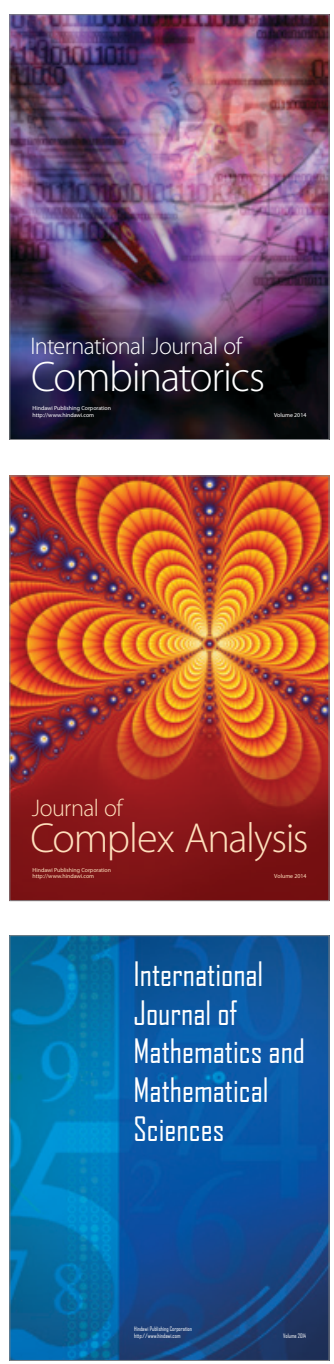
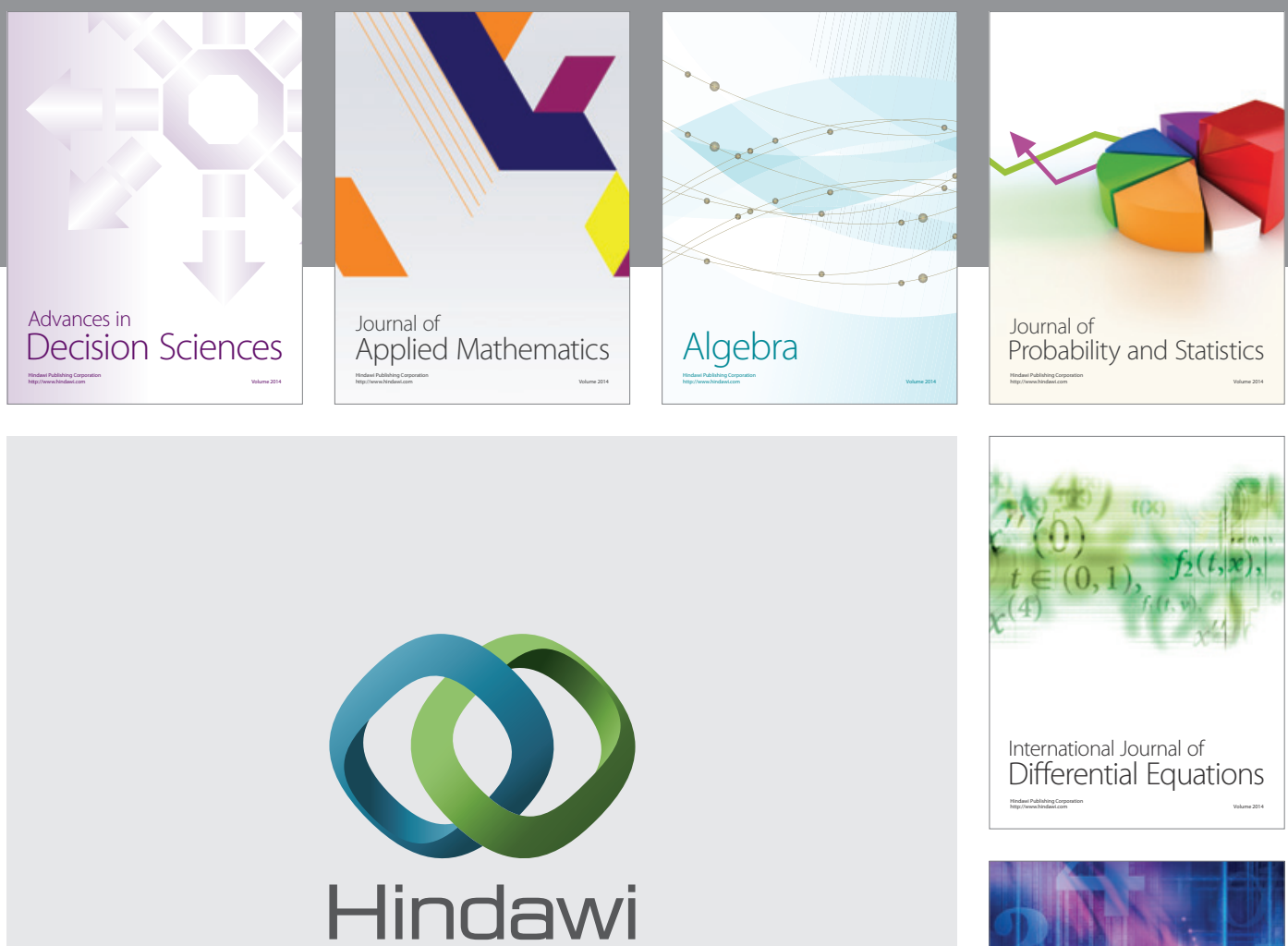

Submit your manuscripts at http://www.hindawi.com
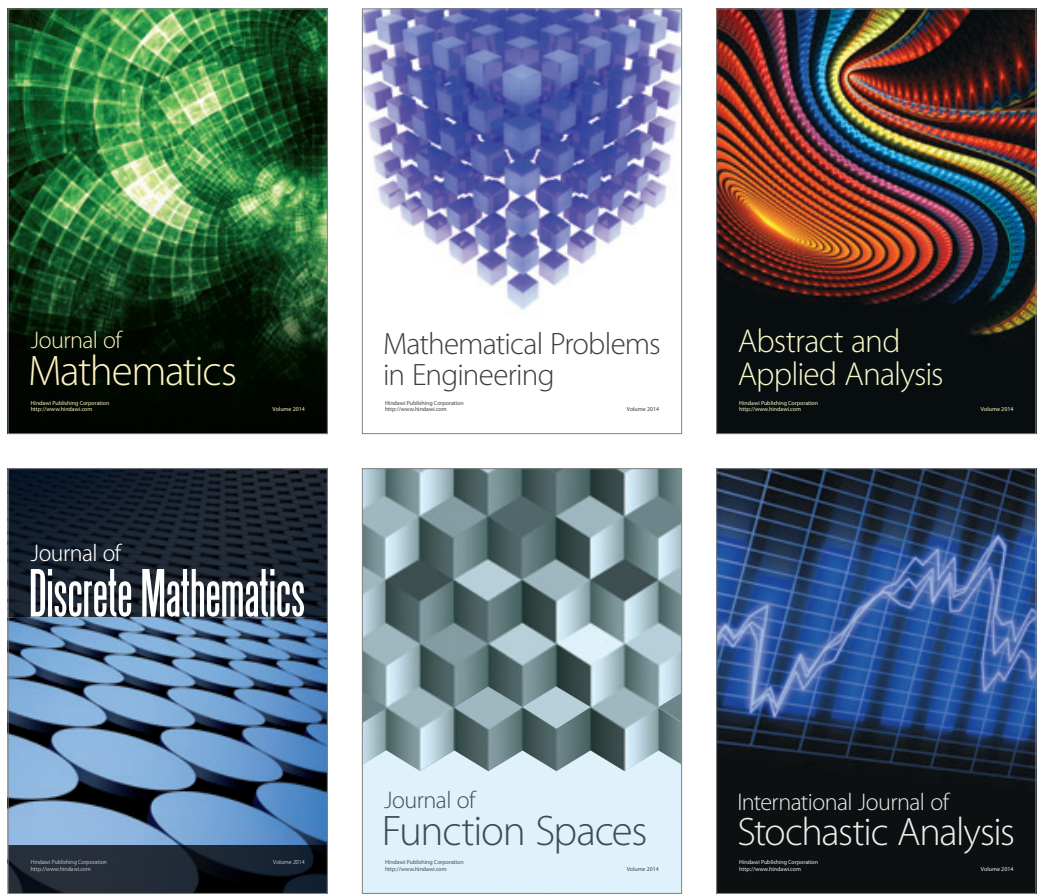

Journal of

Function Spaces

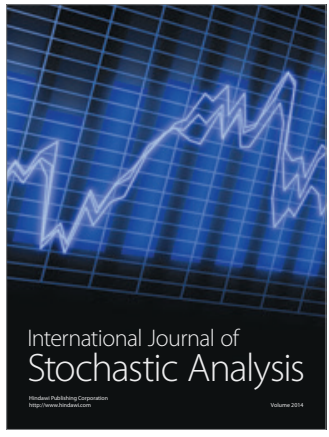

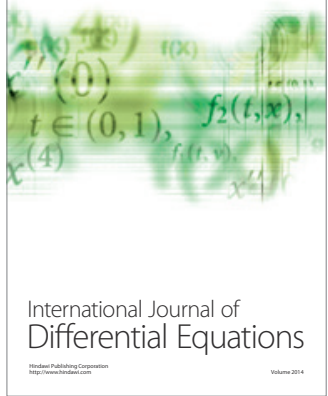
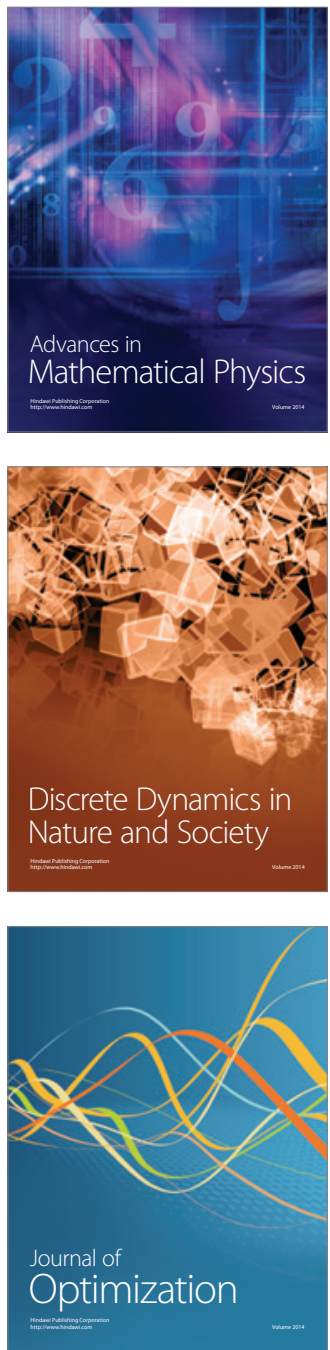